\title{
Scaled Second Order Perturbation Corrections to Configuration Interaction Singles: Efficient and Reliable Excitation Energy Methods
}

\author{
Young Min Rhee and Martin Head-Gordon ${ }^{*}$ \\ Department of Chemistry, University of California and \\ Chemical Sciences Division, Lawrence Berkeley National Laboratory, \\ Berkeley, CA 94720
}

\begin{abstract}
Two modifications of the perturbative doubles correction to configuration interaction with single substitutions (CIS(D)) are suggested, which are excited state analogs of ground state scaled second order Møller-Plesset (MP2) methods. The first approach employs two parameters to scale the two spin components of the direct term of CIS(D), starting from the two-parameter spin-component scaled (SCS) MP2 ground state, and is termed SCS-CIS(D). An efficient resolution-of-the-identity (RI) implementation of this approach is described. The second approach employs a single parameter to scale only the opposite-spin direct term of CIS(D), starting from the one-parameter scaled opposite spin (SOS) MP2 ground state, and is called SOS-CIS(D). By utilizing auxiliary basis expansions and a Laplace transform, a fourth order algorithm for SOS-CIS(D) is described and implemented. The parameters describing SCS-CIS(D) and SOS-CIS(D) are optimized based on a training set including valence excitations of various organic molecules and Rydberg transitions of water and ammonia, and they significantly improve upon CIS(D) itself. The accuracy of the two methods is found to be comparable. This

\footnotetext{
* To whom correspondence should be addressed. E-mail: mhg@cchem.berkeley.edu.
} 
arises from a strong correlation between the same-spin and opposite-spin portions of the excitation energy terms. The methods are successfully applied to the zincbacteriochlorinbacteriochlorin charge transfer transition, for which time-dependent density functional theory, with presently available exchange-correlation functionals, is known to fail. The methods are also successfully applied to describe various electronic transitions outside of the training set. The efficiency of SOS-CIS(D) and the auxiliary basis implementation of CIS(D) and SCS-CIS(D) are confirmed with a series of timing tests. 


\section{Introduction}

Accurate characterization of excited states in large molecules remains a challenge in quantum chemistry. Even though there are highly reliable methods applicable to single- and multi-reference regimes such as equation-of-motion $(\mathrm{EOM})^{1,2}$ or linear response $(\mathrm{LR})^{3-5}$ coupled cluster $(\mathrm{CC})^{6}$ theories and complete active space second order perturbation theory (CASPT2), ${ }^{7}$ they can only be applied to very small systems due to their prohibitively expensive computational cost.

For this practical reason, more efficient and consequently less robust methods are widely used at the present. Various methods have been developed in both electron density-based and wavefunction-based theories. ${ }^{8}$ Time-dependent density functional theory (TDDFT), ${ }^{9,10}$ which uses the response of the electron density to a perturbation from an external electric field (i.e. light), is perhaps the most widely used approach at present. Despite its low cost mean-field level computational effort (formally scaling $\sim N^{4}$ or better with respect to the system size $N$ ), TDDFT has been shown to be reliable for many chemically interesting systems. ${ }^{8}$ However, it has a serious failure in the description of an important class of excitations. ${ }^{11}$ TDDFT calculations must use an approximation for the exchange-correlation (xc) functional, and no xc-functional at present is known to be efficient, reliable for various systems and free from the self-interaction-error. ${ }^{12}$ As a result, TDDFT with the approximate xc-functionals will lead to significant errors for nonlocal electronic transitions such as charge transfer excitations, which are common in large molecules in organic, inorganic and biological chemistry, as well as Rydberg excited states and, very likely, excited states that have very little single excitation character. 
Accordingly, it is natural to look to wavefunction-based alternatives in treating such systems. The most efficient excited state methods that consider electron correlations in this wavefunction-based regime are the CIS(D) ${ }^{13}$ and the approximate second order coupled-cluster (CC2) $)^{14}$ approach. While both approaches have fifth order $\left(\sim N^{5}\right)$ formal scaling of computational cost, CIS(D) is more efficient for the calculation of large molecules because it does not require any time-consuming iterative search for the excitation amplitudes. Nevertheless, the major drawback in applying CIS(D) is still its cost compared to TDDFT. The formal scaling of CIS(D) is at least one power of system size more demanding than TDDFT (and even worse for large systems), and its prefactor tends to be large with numerous direct/semi-direct evaluations of electron repulsion integrals $^{15,16}$ and their transforms between the atomic and molecular representations. This difficulty is partially remedied with the introduction of the resolution-of-the-identity (RI) approximation ${ }^{17,18}$ (or often termed as “density fitting” approximation ${ }^{19,20}$ ), which significantly reduces the size of the prefactor. ${ }^{21,22}$ However, the formal $\left(\sim N^{5}\right)$ scaling cannot be changed with the RI approximation, and RI-CIS(D) will still always be significantly slower than TDDFT for calculations of large molecules.

In this article, we revisit CIS(D) theory with a detailed inspection of the expressions for its spin components, and their contributions to excitation energies. By individually scaling the same-spin and opposite-spin components of CIS(D) terms, we show that a systematic improvement can be obtained relative to CIS(D) itself. We call this approach the spin component scaled (SCS) CIS(D) method, as it is a natural generalization of the corresponding ground state SCS-MP2 method. ${ }^{23}$ We also show that a similar systematic improvement is achieved by using only the opposite-spin 
components as was also shown to be the case for the MP2 ground state. ${ }^{24}$ An additional, and more important benefit of using this scaled opposite-spin (SOS) approach over SCSCIS(D) is its improved efficiency $\left(\sim N^{4}\right.$ as opposed to $\left.\sim N^{5}\right)$ through the use of a Laplace transform. $^{25,26}$ This low-scaling characteristic allows SOS-CIS(D) to be applied to calculations on larger molecules than CIS(D) itself.

As already alluded to above, scaling of spin components is by no means a new concept. The idea was originated by Grimme who reported that the ground state energy of second order Møller-Plesset perturbation theory (MP2) can be systematically improved by separate scaling of same-spin and opposite-spin contributions to the correlation energies. ${ }^{23}$ This spin-component scaling scheme of the MP2 excitation amplitudes was later also applied to CIS(D), though only on the so-called indirect term (see next section), and some improvement was reported in the accuracy of low-lying valence excitation energy predictions. ${ }^{27}$ For ground state MP2, Jung et al. further developed this scaling idea by demonstrating that similar improvement can be attained with only the opposite spin component, ${ }^{24}$ while computational effort can be reduced from $N^{5}$ to $N^{4}$. The present work is a natural extension of these scaling ideas to excited state theories.

The remainder of this paper is arranged as follows. In Sec. II, we develop expressions for the SCS- and SOS-CIS(D) theories starting from the conventional CIS(D) method and its RI-approximated algorithm. These theories are developed in close relationship with their ground state counterparts (SCS- and SOS-MP2), ${ }^{23,24}$ from which the empirical scaling factors can be directly transferred to the indirect term of CIS(D), which depends on ground state pair correlations. During the development, additional empirical parameters are introduced for spin component scaling of the direct term, which 
contains excited-state-specific pair correlations, to recover the total correlation effect on the excitation energies. For SOS-CIS(D) theory, the equations are further developed to permit the implementation of an efficient fourth order algorithm. In Sec. III, the empirical parameters for the direct terms are determined by using various valence transitions of organic molecules adopted by Grimme et $a l^{27}$ and experimentally wellcharacterized Rydberg transitions of water and ammonia. In Sec. IV, numerical tests are performed for the proposed methods. First, it is also shown that such parameters present systematic improvements over conventional CIS(D) in terms of the mean absolute errors in the excitation energies for both SCS-CIS(D) and SOS-CIS(D). More importantly, it is shown that the new methods can present a balanced description between valence and Rydberg transitions, which has not been attained with either conventional CIS(D) or TDDFT using standard functionals. Additionally, it is shown that the present method indeed is adequate in describing a well-known charge transfer transition, which is again not qualitatively correct using TDDFT with common functionals. Finally, we describe the computational cost associated with the methods, and show that SOS-CIS(D) is applicable to large systems with more than 100 heavy atoms. Concluding remarks follow in the last section of the paper.

\section{Theory}

In the following equations, $i, j, \ldots$ and $a, b, \ldots$ will represent occupied and virtual spin-orbitals, whereas $p, q, \ldots$ will denote both occupied and virtual orbitals. When different spins have to be distinguished, we will use $\bar{i}, \bar{j}, \ldots, \bar{a}, \bar{b}, \ldots$ to represent orbitals in the $\beta$-space. Because the distinctions between spin orbital equations and pure spatial 
orbital equations are self-explanatory, the use of $i, j, \ldots, a, b, \ldots$ for spatial orbitals in the $\alpha$-space will not pose any ambiguity. In addition, we will use $R, S, \ldots$ to denote the auxiliary basis functions for the RI approximation. When designating the computational costs, $O / V / N / X$ will be used to represent the numbers of occupied molecular orbitals (MOs), virtual MOs, basis functions, and the corresponding auxiliary basis functions, respectively.

A. CIS(D) Theory. CIS(D) theory was designed to improve upon the intuitively hypothesized CIS-MP2 method. ${ }^{28}$ However, it can also be derived as a truncated solution $^{13,29}$ of rigorous linear response coupled cluster theory. ${ }^{30}$ For completeness, we briefly overview the CIS(D) method below.

When the Hartree-Fock ground state of a system is described by a single determinant $\Phi_{0}$ and when its single substitutions of any occupied spin orbital $i$ to any unoccupied spin orbital $a$ is denoted as $\Phi_{i}^{a}$, the CIS excitation energy $\omega$ is obtained as the solution to an eigenvalue equation

$$
\left\langle\Phi_{i}^{a}|\bar{H}| U_{1} \Phi_{0}\right\rangle=\omega b_{i}^{a},
$$

where $\bar{H}=H-E_{\mathrm{HF}}$ and $U_{1}$ is an operator that generates the CIS wavefunction from $\Phi_{0}$

$$
\Phi_{\mathrm{CIS}}=U_{1} \Phi_{0}=\sum_{i a} b_{i}^{a} \Phi_{i}^{a}
$$

The correlation energy of the excited state corrected through second-order perturbative theory is then given by ${ }^{13,29}$

$$
E^{\mathrm{CIS}(\mathrm{D})}=\left\langle\Phi_{\mathrm{CIS}}|V| U_{2} \Phi_{0}\right\rangle+\left\langle\Phi_{\mathrm{CIS}}|V| T_{2} U_{1} \Phi_{0}\right\rangle,
$$


where $V$ is the fluctuation potential due to electron correlation, and $T_{2}$ is the operator that generates the first-order Møller-Plesset wavefunction from $\Phi_{0}$

$$
\begin{aligned}
T_{2} \Phi_{0} & =\frac{1}{4} \sum_{i j a b} a_{i j}^{a b} \Phi_{i j}^{a b} \\
& =-\frac{1}{4} \sum_{i j a b} \frac{(i j \| a b)}{\varepsilon_{a}+\varepsilon_{b}-\varepsilon_{i}-\varepsilon_{j}} \Phi_{i j}^{a b} .
\end{aligned}
$$

$U_{2}$ is the operator that generates the first order excited state pair correlations:

$$
\begin{aligned}
U_{2} \Phi_{0} & =\frac{1}{4} \sum_{i j a b} b_{i j}^{a b} \Phi_{i j}^{a b} \\
& =-\frac{1}{4} \sum_{i j a b} \frac{\left\langle\Phi_{i j}^{a b}|V| U_{1} \Phi_{0}\right\rangle}{\varepsilon_{a}+\varepsilon_{b}-\varepsilon_{i}-\varepsilon_{j}-\omega} \Phi_{i j}^{a b} .
\end{aligned}
$$

Physically, the first term in eq 3 (the "direct" term) accounts for electron correlation effects that involve one electron that is active in the CIS excitation plus a second electron, thereby generating double excitations. The second term (which we will refer to as the "indirect" term) accounts for the effect of electron correlations between pairs of electrons that are not directly involved in the CIS excitation - which is why it involves the product of the ground state doubles amplitudes with the CIS amplitudes. After a little algebra, it can be shown that eq 3 can be transformed into

$$
\begin{aligned}
E^{\mathrm{CIS}(\mathrm{D})}-E^{\mathrm{MP} 2}= & -\frac{1}{4} \sum_{i j a b} \frac{\left(u_{i j}^{a b}\right)^{2}}{\varepsilon_{a}+\varepsilon_{b}-\varepsilon_{i}-\varepsilon_{j}-\omega} \\
& +\sum_{i a b} b_{i}^{a} b_{i}^{b} R_{a b}+\sum_{i j c} b_{i}^{c} b_{j}^{c} R_{i j}+\sum_{i a} b_{i}^{a} w_{i}^{a}
\end{aligned}
$$

with the following definitions:

$$
\begin{aligned}
& u_{i j}^{a b}=\sum_{c}\left[(a b \| c j) b_{i}^{c}-(a b \| c i) b_{j}^{c}\right]+\sum_{k}\left[(k a \| i j) b_{k}^{b}-(k b \| i j) b_{k}^{a}\right], \\
& R_{a b}=-\sum_{j k b c}(j c \mid k b) a_{j k}^{c a},
\end{aligned}
$$




$$
\begin{aligned}
& R_{i j}=-\sum_{j k a b}(j a \mid k b) a_{i k}^{a b}, \\
& w_{i}^{a}=\sum_{j k b c}(j k \| b c) a_{i k}^{a c} b_{j}^{b} .
\end{aligned}
$$

Eq 6 defines the second-order correction to the CIS excitation energy, $\omega^{\mathrm{CIS(D)}}$, leading a total excitation energy that is $\omega^{\mathrm{CIS}}+\omega^{\mathrm{CIS}(\mathrm{D})}$.

B. Resolution-of-the-identity in CIS(D) Theory. Let us first introduce the auxiliary basis as a resolution-of-the-identity (RI) approximation in the CIS(D) theory. The RI approximation describes all electron-repulsion integrals (ERIs) in eqs 7 - 10 as

$$
\begin{aligned}
(p q \mid r s)_{\mathrm{RI}} & =\sum_{P Q}(p q \mid P)(P \mid Q)^{-1}(Q \mid r s) \\
& =\sum_{R} B_{p q}^{R} B_{r s}^{R},
\end{aligned}
$$

with the $\mathbf{B}$ matrix defined as

$$
B_{p q}^{R}=\sum_{P}(p q \mid P)(P \mid R)^{-1 / 2}
$$

From a computational point of view, it is advantageous to define three other related quantities:

$$
\begin{aligned}
& V_{a i}^{R}=\sum_{b} b_{i}^{b} B_{a b}^{R}, \\
& O_{a i}^{R}=\sum_{j} b_{j}^{a} B_{i j}^{R}, \\
& D_{a i}^{R}=V_{a i}^{R}-O_{a i}^{R} .
\end{aligned}
$$

With these definitions, it is easy to show that eq 7 can be transformed into

$$
u_{i j}^{a b}=\sum_{P}\left[D_{a i}^{P} B_{b j}^{P}-D_{b i}^{P} B_{a j}^{P}+B_{a i}^{P} D_{b j}^{P}-B_{b i}^{P} D_{a j}^{P}\right] .
$$


Eqs 8 - 10 can be obtained by combining the ERI $(i a \mid j b)_{\mathrm{RI}}$ and the amplitude $a_{i j}^{a b}$.

An efficient algorithm for this resolution of the identity formulation of CIS(D) theory is presented in Figure 1, where one can easily see that its cost scales with the fifth power of system size. In addition, the disk transfer cost is fourth order, with the size of the storage space requirement scaling as third order. In this algorithm, a batching scheme is introduced to minimize the cost for disk input-output (I/O), especially for $D_{a i}^{R}$ (Line 4). The I/O cost decreases with a larger batch size, and the maximum batch size can be easily calculated from the size of available memory and disk space. One important point is that for a calculation of $S$ excited states, the total cost grows as $2 O^{2} V^{2} X S$. The algorithm possesses three additional fifth order steps related to the computation of $(i a \mid j b)_{\mathrm{RI}}, R_{a b}$, and $R_{i j}$, but these do not depend on $S$.

It is interesting to note the possibility of a minor modification of the above algorithm. Based on the formal similarity to RI-MP2 gradient theory, ${ }^{31,32}$ eqs 8 and 9 can be rearranged as

$$
\begin{aligned}
& R_{a b}=\sum_{k P} B_{b k}^{P} \Gamma_{a k}^{P}, \\
& R_{i j}=\sum_{a P} B_{a j}^{P} \Gamma_{a i}^{P},
\end{aligned}
$$

where, following RI-MP2 gradient theory, the three-center two-particle density matrix is:

$$
\Gamma_{a i}^{P}=-\sum_{j b Q} a_{i j}^{a b}(j b \mid Q)(P \mid Q)^{-1 / 2}
$$

This leads to an alternative working expression for $w_{i}^{a}$ as well:

$$
w_{i}^{a}=-\sum_{j b P} B_{b j}^{P} \Gamma_{a i}^{P} b_{j}^{b}-\sum_{j k b c}(j c \mid k b) a_{i k}^{a c} b_{j}^{b} .
$$


The potential benefit of using $\Gamma_{a i}^{P}$ will be reduced disk IO cost. While eqs 8 and 9 require fourth order disk IO related to the storage of ERIs and the $a$-amplitudes (Line 17 and 18 in Figure 1), the use of $\Gamma_{a i}^{P}$ would only require third order disk access for the calculations of $R_{a b}$ and $R_{i j}$. However, the CPU cost of calculating eq 19 will be $O^{2} V^{2} X$. In fact, this is larger than the combined cost of eqs 8 and $9\left(O^{2} V^{2} N\right)$. This additional CPU time will become more important as the system size grows. Therefore, it is more desirable to generate $R_{a b}$ and $R_{i j}$ based on the RI-approximated integrals without using $\Gamma_{a i}^{P}$. However, the use of $\Gamma_{a i}^{P}$ will be crucial in the efficient implementation of the SOSCIS(D) theory as will be shown later.

C. The SCS-CIS(D) Method. We define SCS-CIS(D) theory in an analogy to the manner in which Grimme first proposed the corresponding ground state SCS-MP2 method, by scaling the same-spin and opposite-spin components of the energy. In addition to this split spin component treatment, an empirical damping factor $0 \leq \lambda \leq 1$ for the CIS excitation energy $\omega$ is introduced for the direct term as

$$
\begin{aligned}
& U_{2}^{\mathrm{SS}} \Phi_{0}=-\frac{1}{4} \sum_{i j a b} \frac{\left\langle\Phi_{i j}^{a b}|V| U_{1} \Phi_{0}\right\rangle}{\varepsilon_{a}+\varepsilon_{b}-\varepsilon_{i}-\varepsilon_{j}-\lambda \omega} \Phi_{i j}^{a b}-\frac{1}{4} \sum_{i j \bar{a} \bar{b}} \frac{\left\langle\Phi_{\bar{i} \bar{j}}^{\bar{a} \bar{b}}|V| U_{1} \Phi_{0}\right\rangle}{\varepsilon_{\bar{a}}+\varepsilon_{\bar{b}}-\varepsilon_{\bar{i}}-\varepsilon_{\bar{j}}-\lambda \omega} \Phi_{\overline{i j}}^{\bar{a} \bar{b}} \\
& U_{2}^{\mathrm{OS}} \Phi_{0}=-\sum_{\bar{i} \bar{a} \bar{b}} \frac{\left\langle\Phi_{\bar{j}}^{\bar{a} b}|V| U_{1} \Phi_{0}\right\rangle}{\varepsilon_{\bar{a}}+\varepsilon_{b}-\varepsilon_{\bar{i}}-\varepsilon_{j}-\lambda \omega} \Phi_{\overline{i j}}^{\bar{a} b} .
\end{aligned}
$$

We will detail the role of $\lambda$ in a later section. With the obvious components of the indirect term as in SCS-MP2, the spin-component scaling modification of eq 3 will become: 


$$
\begin{aligned}
E^{\mathrm{SCS}-\mathrm{CIS}(\mathrm{D})}= & \left\langle\Phi_{\mathrm{CIS}}|V|\left(c_{U}^{\mathrm{OS}} U_{2}^{\mathrm{OS}}+c_{U}^{\mathrm{SS}} U_{2}^{\mathrm{SS}}\right) \Phi_{0}\right\rangle \\
& +\left\langle\Phi_{\mathrm{CIS}}|V|\left(c_{T}^{\mathrm{OS}} T_{2}^{\mathrm{OS}}+c_{T}^{\mathrm{SS}} T_{2}^{\mathrm{SS}}\right) U_{1} \Phi_{0}\right\rangle .
\end{aligned}
$$

With the independent scaling of $U_{2}$-term and the use of the damping factor $(\lambda)$, this equation differs significantly from a previous suggestion for defining SCS-CIS(D), ${ }^{27}$ which left the first term of eq 3 unmodified, but replaced the second term as we have done in the above.

When the ground state correlation energy contribution is separated as in eq 6, the SCS correlation correction to the CIS energy can be written as

$$
\omega^{\mathrm{SCS}-\mathrm{CIS}(\mathrm{D})}=c_{U}^{\mathrm{OS}} w_{U}^{\mathrm{OS}}+c_{T}^{\mathrm{OS}} w_{T}^{\mathrm{OS}}+c_{U}^{\mathrm{SS}} w_{U}^{\mathrm{SS}}+c_{T}^{\mathrm{SS}} w_{T}^{\mathrm{SS}},
$$

with the obvious definition for each of the terms. In practice, the opposite-spin (OS) and the same-spin (SS) component splitting of the $U$ operator can be performed without any additional computational cost during the first summation in eq 6 . In contrast, the splitting of the $T$ operator requires separate evaluations of OS and SS contributions to $R_{a b}, R_{i j}$, and $w_{i}^{a}$. In closed-shell systems, this is attained at an additional cost of $O^{2} V^{2} N$ (eqs 8 and 9). However, compared to the leading cost of $2 O^{2} V^{2} X S$, this additional cost of SCSCIS(D) is negligible especially when excitation energies to multiple states are calculated at the same time.

D. The SOS-CIS(D) Theory. The opposite spin part of the CIS(D) correction can be extracted from eq 22 as

$$
E^{\mathrm{OS}-\mathrm{CIS}(\mathrm{D})}=\left\langle\Phi_{\mathrm{CIS}}|V| U_{2}^{\mathrm{OS}} \Phi_{0}\right\rangle+\left\langle\Phi_{\mathrm{CIS}}|V| T_{2}^{\mathrm{OS}} U_{1} \Phi_{0}\right\rangle .
$$


From the symmetry of $u_{i j}^{a b}$ with respect to the permutation of indices, it is easy to show that the first term in this equation becomes

$$
\left\langle\Phi_{\mathrm{CIS}}|V| U_{2}^{\mathrm{OS}} \Phi_{0}\right\rangle=-\sum_{\overline{i j} \bar{a} b} \frac{\left(u_{\bar{i} j}^{\bar{a} b}\right)^{2}}{\varepsilon_{\bar{a}}+\varepsilon_{b}-\varepsilon_{\bar{i}}-\varepsilon_{j}-\lambda \omega},
$$

where we again use the empirical damping factor $0 \leq \lambda \leq 1$, and have

$$
u_{\bar{i} \bar{b}}^{\bar{a} b}=\sum_{P}\left[D_{\bar{a} \bar{i}}^{P} B_{b j}^{P}+B_{\bar{a} \bar{i}}^{P} D_{b j}^{P}\right]
$$

Likewise, the second term in eq 24 can be expressed as

$$
\begin{aligned}
\left\langle\Phi_{\mathrm{CIS}}|V| T_{2}^{\mathrm{OS}} U_{1} \Phi_{0}\right\rangle= & E^{\mathrm{OS}-\mathrm{MP} 2}+\sum_{i a b} b_{i}^{a} b_{i}^{b} R_{a b}+\sum_{\overline{\bar{a}} \bar{b}} b_{\bar{i}}^{\bar{a}} b_{\bar{i}}^{\bar{b}} R_{\bar{a} \bar{b}} \\
& +\sum_{i j c} b_{i}^{c} b_{j}^{c} R_{i j}+\sum_{\overline{i j} \bar{c}} b_{\bar{i}}^{\bar{c}} b_{\bar{j}}^{\bar{c}} R_{\overline{i j}}+\sum_{i a} b_{i}^{a} w_{i}^{a}+\sum_{\bar{i} \bar{a}} b_{\bar{i}}^{\bar{a}} w_{\bar{i}}^{\bar{a}},
\end{aligned}
$$

with

$$
\begin{aligned}
& R_{a b}=-\sum_{\overline{j k} \bar{c}}(\overline{j \bar{c}} \mid k b) a_{\overline{j k}}^{\bar{c} a}, \\
& R_{i j}=-\sum_{\bar{k} a \bar{b}}(j a \mid \overline{k b}) a_{i \bar{k}}^{a \bar{b}}, \\
& w_{i}^{a}=\sum_{j \bar{k} b \bar{c}}(j b \mid \bar{k} \bar{c}) a_{i \bar{k}}^{a \bar{c}} b_{j}^{b}+\sum_{\overline{j k b \bar{c}}}(\overline{j k} \| \bar{b} \bar{c}) a_{i \bar{k}}^{a \bar{c}} b_{\bar{j}}^{\bar{b}},
\end{aligned}
$$

The beta spin intermediates, $R_{\bar{a} \bar{b}}, R_{\overline{i j}}$, and $w_{\bar{i}}^{\bar{a}}$ are defined analogously. Also, $E^{\text {OS-MP2 }}$ denotes the opposite spin component of MP2 correction. $^{24}$

This opposite-spin formalism can be transformed into a fourth order algorithm ${ }^{24}$ through the use of a Laplace transform with discrete numerical quadratures ${ }^{26}$

$$
x^{-1}=\int_{0}^{\infty} d t \exp (-x t)=\sum_{t} \rho_{t} \exp (-x t)
$$

in conjunction with the RI approximation. Firstly, eq 25 can be transformed as 


$$
\begin{aligned}
w_{\mathrm{I}} & =-\sum_{t} \rho_{t} \sum_{P Q} \sum_{\bar{j} \bar{a} \bar{a} b}\left[D_{\bar{a} \bar{i}}^{P} B_{b j}^{P}+B_{\bar{a} \bar{i}}^{P} D_{b j}^{P}\right]\left[D_{\bar{a} \bar{i}}^{Q} B_{b j}^{Q}+B_{\bar{a} \bar{i}}^{Q} D_{b j}^{Q}\right] e^{-\left(\varepsilon_{\bar{a}}+\varepsilon_{b}-\varepsilon_{\bar{i}}-\varepsilon_{j}-\lambda \omega\right) t} \\
& =-\sum_{t} \rho_{t} \sum_{P Q} \sum_{\bar{i} \bar{j} \bar{a} b}\left[D_{\bar{a} \bar{i}}^{P} B_{b j}^{P}+B_{\bar{a} \bar{i}}^{P} D_{b j}^{P}\right]\left[\tilde{D}_{\bar{a} \bar{i}}^{Q} \tilde{B}_{b j}^{Q}+\tilde{B}_{\bar{a} \bar{i}}^{Q} \tilde{D}_{b j}^{Q}\right] e^{\lambda \omega t},
\end{aligned}
$$

with $\tilde{B}_{a i}^{R}=B_{a i}^{R} e^{\left(\varepsilon_{i}-\varepsilon_{a}\right) t}$ and $\tilde{D}_{a i}^{R}=D_{a i}^{R} e^{\left(\varepsilon_{i}-\varepsilon_{a}\right) t}$. Let us denote terms from eq 27 involving $R_{i j}$ and $R_{a b}$ as $w_{\text {II }}$ :

$$
w_{\mathrm{II}}=\sum_{i a b} b_{i}^{a} b_{i}^{b} R_{a b}+\sum_{\bar{i} \overline{\bar{a}} \bar{b}} b_{\bar{i}}^{\bar{a}} b_{\bar{i}}^{\bar{b}} R_{\bar{a} \bar{b}}+\sum_{i j c} b_{i}^{c} b_{j}^{c} R_{i j}+\sum_{\bar{i} \bar{c}} b_{\bar{i}}^{\bar{c}} b_{\bar{j}}^{\bar{c}} R_{\overline{i j}} .
$$

In addition, it is easy to show that the last terms in eq 27 that involve $w_{i}^{a}$ can be expressed as

$$
\begin{aligned}
w_{\mathrm{III}}=-\sum_{t} \rho_{t} \times & {\left[\sum_{\bar{k} \bar{c}} \sum_{P Q}\left(f_{P}^{\alpha}+f_{P}^{\beta}\right) \tilde{f}_{Q}^{\alpha} B_{\bar{c} \bar{k}}^{P} \tilde{B}_{\bar{c} \bar{k}}^{Q}-\sum_{\overline{j k c}} \sum_{P Q} B_{\bar{c} \bar{j}}^{P} V_{\bar{j} \bar{k}}^{P} \tilde{B}_{\bar{c} \bar{k}}^{Q} \tilde{f}_{Q}^{\alpha}\right.} \\
& \left.+\sum_{k c} \sum_{P Q}\left(f_{P}^{\alpha}+f_{P}^{\beta}\right) \tilde{f}_{Q}^{\beta} B_{c k}^{P} \tilde{B}_{c k}^{Q}-\sum_{j k c} \sum_{P Q} B_{c j}^{P} V_{j k}^{P} \tilde{B}_{c k}^{Q} \tilde{f}_{Q}^{\beta}\right],
\end{aligned}
$$

with the definitions: $V_{i j}^{R}=\sum_{a} b_{i}^{a} B_{a j}^{R}, f_{R}^{\alpha}=\sum_{a i} b_{i}^{a} B_{a i}^{R}, \tilde{f}_{R}^{\alpha}=\sum_{a i} b_{i}^{a} \tilde{B}_{a i}^{R}$ and their analogs in the $\beta$-spin space.

A fourth order algorithm can be implemented by carefully rearranging the order of summations in various terms. When $\mathbf{X}, \mathbf{Y}$, and $\mathbf{Z}$ are defined as

$$
\begin{aligned}
& X_{P Q}^{\alpha}=\sum_{a i} B_{a i}^{P} \tilde{B}_{a i}^{Q}, \\
& Y_{P Q}^{\alpha}=\sum_{a i} D_{a i}^{P} \tilde{B}_{a i}^{Q}=\sum_{a i} \tilde{D}_{a i}^{P} B_{a i}^{Q}, \\
& Z_{P Q}^{\alpha}=\sum_{a i} D_{a i}^{P} \tilde{D}_{a i}^{Q},
\end{aligned}
$$

together with their obvious $\beta$-spin analogs, the first OS-CIS(D) correction term becomes

$$
w_{\mathrm{I}}=-\sum_{t} \rho_{t} e^{\lambda \omega t} \sum_{P Q}\left(X_{P Q}^{\alpha} Z_{P Q}^{\beta}+Y_{Q P}^{\alpha} Y_{P Q}^{\beta}+Y_{P Q}^{\alpha} Y_{Q P}^{\beta}+Z_{P Q}^{\alpha} X_{P Q}^{\beta}\right) .
$$


Also, in analogy to the RI-CIS(D) case, when $\Gamma_{a i}^{P}$ is introduced as

$$
\Gamma_{a i}^{P}=\sum_{t} \rho_{t} \sum_{Q} \tilde{B}_{a i}^{Q} X_{P Q}^{\beta}
$$

it is trivial to show that eqs 28 and 29 are equivalent to

$$
\begin{aligned}
& R_{a b}=\sum_{i P} \Gamma_{a i}^{P} B_{b i}^{P}, \\
& R_{i j}=\sum_{a P} B_{a j}^{P} \Gamma_{a i}^{P} .
\end{aligned}
$$

Finally, when $\mathbf{G}$ and $\mathbf{H}$ matrices are defined as

$$
\begin{aligned}
& G_{c k}=\sum_{j P} B_{c j}^{P} V_{j k}^{P}, \\
& H_{c k}=\sum_{j P} \tilde{B}_{c k}^{P} \tilde{f}_{P}^{\beta},
\end{aligned}
$$

the last term of the OS-CIS(D) correction becomes

$$
\begin{aligned}
w_{\mathrm{III}}=-\sum_{t} \rho_{t} \times[ & \sum_{P Q}\left(f_{P}^{\alpha}+f_{P}^{\beta}\right) \tilde{f}_{Q}^{\alpha} X_{P Q}^{\beta}-\sum_{\bar{k} \bar{c}} G_{\bar{c} \bar{k}} H_{\bar{c} \bar{k}} \\
& \left.+\sum_{P Q}\left(f_{P}^{\alpha}+f_{P}^{\beta}\right) \tilde{f}_{Q}^{\beta} X_{P Q}^{\alpha}-\sum_{k c} G_{c k} H_{c k}\right] .
\end{aligned}
$$

By collecting the above expressions, the scaled opposite spin CIS(D) excitation energies are obtained as

$$
\begin{aligned}
\omega^{\mathrm{SOS}-\mathrm{CIS}(\mathrm{D})} & =\left\langle\Phi_{\mathrm{CIS}}|V| c_{U} U_{2}^{\mathrm{OS}} \Phi_{0}\right\rangle+\left\langle\Phi_{\mathrm{CIS}}|V| c_{T} T_{2}^{\mathrm{OS}} U_{1} \Phi_{0}\right\rangle \\
& =c_{U} w_{\mathrm{I}}+c_{T}\left(w_{\mathrm{II}}+w_{\mathrm{III}}\right),
\end{aligned}
$$

with two empirical scaling parameters, $c_{U}$ and $c_{T}$, where the latter is already fixed from the ground state SOS-MP2 energy, and the former is to be determined by comparing against either higher accuracy calculations or experiments. 
Of the various working expressions listed in the above, only eqs 36 and 37 need to be evaluated for each excited state and each Laplace quadrature point. Accordingly, the computational cost of this method will be dominated by the evaluation of these two equations, requiring a total of $2 O V X^{2} S T$ operations, with $S$ and $T$ denoting the numbers of excited states and quadrature points, respectively. The resulting overall algorithm is shown in Figure 2. Comparing against the ground state SOS-MP2 method, we conclude that the cost per state (for $S$ not too small) will be approximately twice the cost of the corresponding ground state SOS-MP2 calculation. Also, it should be noted that the Laplace transform in our algorithm does not require any aggressive integral screening scheme, ${ }^{33}$ which is practically required for an efficient treatment of the same-spin component calculation.

\section{Optimizations of Parameters}

As shown in the previous section, the proposed methods require optimization of various parameters. The most straightforward way will be to use experimental data in the determination of these parameters. In this work, the extensive set of organic molecules adopted by Grimme and co-workers ${ }^{27}$ have been used again. This set only includes valence transitions with $\pi \rightarrow \pi^{*}$ and $n \rightarrow \pi^{*}$ characters. To make the training set more complete, we have added well-characterized Rydberg transitions of water and ammonia. (See Table 1 for the complete list of the transitions.)

In the calculations of organic molecules, molecular geometries were obtained at the HF/6-311G(d,p) level for the ground states and the CIS/6-311G(d,p) level for the excited states. These levels of theory are roughly comparable in quality to DFT methods, 
although there is a systematic tendency to make bondlengths slightly too short, and thus vibrational frequencies slightly too high. To obtain 0-0 transition energies, corrections for zero-point energies must be computed for both the ground and the excited states. Frequencies obtained from analytic Hessians at the above levels of theory have been used for this purpose after scaling with a factor of 0.9. In the correlated excitation energy calculations at the optimized geometries, the aug-cc-pVTZ basis ${ }^{34}$ was employed together with its corresponding auxiliary basis set. ${ }^{35}$ The CIS and HF components of the calculation were performed without the RI approximation.

In the case of Rydberg states, 0-0 transitions may not be experimentally observable ${ }^{36}$ because of potentially large Franck-Condon shifts. Accordingly, we have used vertical excitation energies ${ }^{37,38}$ for these transitions. In the excitation energy calculations, we have used 6-311(2+,2+)G(d,p) basis together with the auxiliary basis of aug-cc-pVTZ. ${ }^{34}$ Even though this auxiliary basis was not specifically optimized for the Pople-style basis, the RI approximation error with the basis was always found to be smaller than $0.001 \mathrm{eV}$, similar to the report for the ground state energy calculations. ${ }^{31}$ All calculations were performed with a development version of Q-Chem 3.0. ${ }^{39}$

A. Performance of RI-CIS(D). Because the present SCS- and SOS-CIS(D) theories are based on CIS(D), it is natural to look to the performance of this method to get insight for possible improvements toward SCS- and SOS-CIS(D). Figure 3 presents the errors (against the experimental values) of RI-CIS(D) for the molecules in the training set. Firstly, one can clearly see that there exists a systematic overestimation in the valence transitions: the method tends to give larger transition energies than experiment as 
represented by its mean signed error (MSE) of $0.19 \mathrm{eV}$ for these transitions. In addition, but more notably, the method tends to severely underestimate the transition energies of the Rydberg transitions. This underestimation is indeed a generic problem of the method. As the energy denominator in eq 5 becomes smaller, the magnitude of the direct term becomes larger. Because the correlation correction from the direct term is always negative, an over-correction caused by small denominator (or large $\omega^{\mathrm{CIS}}$ ) leads to this tendency of underestimation in the total transition energies. This effect predominantly appears for Rydberg transitions because qualitatively they involve the lowest lying (most diffuse) virtual orbitals, and therefore the smallest energy denominators which are most sensitive to $\omega$. When the new SCS- and SOS-CIS(D) methods were applied with $\lambda=1$ (no damping), a similar defect was observed for these Rydberg transitions in the training set.

This defect of an unbalanced description of valence and Rydberg excited states will be removed as one introduces higher correction terms of the coupled cluster theory. (Recall that CIS(D) may be expressed as a low order truncated solution of linear response coupled cluster theory.) However, such an approach is not a realistic option for our present work, where the design of an efficient algorithm is under pursuit. Instead, we recall that the problem is mostly remedied when the excitation energy is iteratively calculated in the quasi-degenerate variant $\operatorname{CIS}\left(\mathrm{D}_{0}\right)$ theory, ${ }^{29}$ where $\omega$ is omitted in the calculation of the excitation amplitudes $b_{i j}^{a b}$ (eq 5). The more balanced behavior of CIS $\left(D_{0}\right)$ theory is one motivation for the introduction of the empirical damping factor $\lambda$. A closely related motivation is the above discussion of the difference in the important 
virtual orbitals between Rydberg excited states (low-lying) and valence states (higher lying antibonding orbitals).

We stress that the use of the damping factor is likely to most improve the CIS(D) method when it is combined with spin component scaling. Because the effect of damping will be to decrease the direct correlation correction (in other words, the $U_{2}$-term will become less negative), it will tend to degrade the performance of CIS(D) for valence transitions. We aim to compensate this potential problem through the use of scaling parameters.

In theory, we can test the behavior of both SCS- and SOS-CIS(D) as a function of the $\lambda$ value. Because different $\lambda$ values affect every individual component of the direct term in a different manner (Line 13 in Figure 1), such a test will require tremendous computational effort in the SCS-CIS(D) case. In the SOS-CIS(D) case, however, only one set of calculations can be used to obtain excitation energies at all different $\lambda$ (Line 9 in Figure 2). For this practical reason, we will only use SOS-CIS(D) to obtain the optimal value of the damping factor.

B. Numerical Quadratures for Laplace Transform. For the SOS-CIS(D) method, we need to define the quadrature scheme used to evaluate the Laplace transform. Here, we employed the same scheme previously reported with SOS-MP2 theory, ${ }^{24}$ the ground state counterpart of SOS-CIS(D). Specifically, 10 numerical quadrature points were obtained by minimizing the integrated error

$$
\delta^{2}=\int_{x_{\min }}^{x_{\max }} d x\left(\frac{1}{x}-\sum_{t} \rho_{t} \exp (-x t)\right)^{2}
$$


according to Wilson and Almlöf ${ }^{40}$ with $x_{\min }=0.01$ and $x_{\max }=400$ a.u. The $x=\varepsilon_{a}+\varepsilon_{b}-\varepsilon_{i}-\varepsilon_{j}-\lambda \omega^{\mathrm{CIS}}$ values for all the molecules tested in this work actually fell in this range for all possible $\lambda$ values $(0 \leq \lambda \leq 1)$. The contribution to the excitation energy after the seventh quadrature point was found to be negligible (less than $0.001 \mathrm{eV}$ ) in all test results as was found previously for the ground state case. ${ }^{24}$ Because the contribution from the seventh quadrature point appeared to be considerably smaller than the overall uncertainty level of SOS-CIS(D) (discussed later), one might consider a reduction in the number of quadrature points to improve the efficiency. To preserve the consistency with the ground state description, however, we did not try this in the present work.

In fact, the above scheme will not be the most efficient strategy for the numerical integration of the Laplace transform. The best accuracy with the least number of points is expected if the points and weights are actually determined for the given system, ${ }^{40}$ potentially with two separate quadrature schemes for the direct term and the indirect term. In addition, different quadrature schemes ${ }^{33,41}$ may further reduce the computational cost. We do not consider such possibilities in this work for the following reasons. First, system-specific optimal quadrature points will surely depend on the energy eigenvalues of canonical molecular orbitals and potentially on CIS excitation energies when $\lambda$ is nonzero. This dependency will introduce an undesirable complication when analytic gradients of the ground and excited state surfaces are considered. ${ }^{42}$ Also, using different quadratures for the direct and the indirect terms will result in different $\tilde{B}$ matrices in the terms, increasing the associated computational cost. For the time being, our present approach based on simple least-square Gaussian quadrature achieves sufficient accuracy and efficiency ( $0.001 \mathrm{eV}$ error with only seven quadrature points). 
C. Determination of Damping Factor. Now that the quadrature scheme is defined, we can determine the optimal damping factor as follows. For any given $\lambda$ value, SOS-CIS(D) method has two adjustable empirical parameters. Of the two, the parameter related to the indirect term with the $T_{2}$ operator will be transferred from the counterpart ground state theory (SOS-MP2) for consistency (namely, $c_{T}=1.3$ ). ${ }^{24}$ This leaves only the parameter related to the $U_{2}$ operator. From eq 45 , we can obtain $c_{U}$ according to

$$
c_{U} w_{\mathrm{I}} \approx \omega^{\mathrm{exp}}-\omega^{\mathrm{CIS}}-c_{T}\left(w_{\mathrm{II}}+w_{\mathrm{III}}\right) .
$$

Here, $\omega^{\text {exp }}$ denotes the experimental excitation energies. The root-mean-square (RMS) error of the fit, $\delta(\lambda)$, can be used as an indicator of the fidelity of SOS-CIS(D) as a function of $\lambda$.

Figure 4 shows this RMS error based on the reference transitions at various damping factors. It is interesting to see that the optimal $\lambda$ value is obtained as zero. In fact, this finding is in accordance with the result of CIS( $\left(\mathrm{D}_{0}\right)$ : removing $\omega$ from eq 5 leads to a more balanced description between valence and Rydberg transitions. This complete damping will have another advantage from a mathematical point of view. The Laplace transform in eq 31 is only valid when the denominator $x$ is positive definite. With the complete damping, the lower bound of $x$ becomes equal to twice the HOMO-LUMO gap, which is positive semi-definite, just as in ground state SOS-MP2 theory.

D. Determination of Scaling Parameters. With the determination of the damping factor shown in the above, the scaling parameter of SOS-CIS(D) has been 
already obtained as $c_{U}=1.51$ with an RMS fit error of $0.17 \mathrm{eV}$. The four adjustable parameters of SCS-CIS(D) can be determined in a similar fashion at the given damping factor $(\lambda=0)$. The parameters related to the indirect terms with the $T_{2}$ operator will be again transferred from the counterpart ground state theory (SCS-MP2). For the parameterization, we can use a divariate regression approach to determine the best fit values of $c_{U}^{\mathrm{oS}}$ and $c_{U}^{\mathrm{SS}}$ based on eq 23:

$$
c_{U}^{\mathrm{OS}} w_{U}^{\mathrm{OS}}+c_{U}^{\mathrm{SS}} w_{U}^{\mathrm{SS}} \approx \omega^{\mathrm{exp}}-\omega^{\mathrm{CIS}}-\left(c_{T}^{\mathrm{OS}} w_{T}^{\mathrm{OS}}+c_{T}^{\mathrm{SS}} w_{T}^{\mathrm{SS}}\right)
$$

together with the ground state parameters $c_{T}^{\text {os }}=6 / 5$ and $c_{T}^{\text {SS }}=1 / 3$ from SCS-MP2. ${ }^{23}$ With the electronic transitions listed in Table 1 , the parameters are obtained as $c_{U}^{\mathrm{os}}=1.67$ and $c_{U}^{\text {sS }}=-0.36$ with an RMS fit error of $0.17 \mathrm{eV}$.

At first, it may be surprising that this fit leads to an unphysical negative scaling parameter. Moreover, RMS fit error from SCS-CIS(D) is practically the same as in SOSCIS(D) even though there are more fitting parameters in this case. However, this is quite understandable from the dependence of the same-spin and opposite-spin components of both direct and indirect terms as shown in Figure 5. Because there is a strong correlation between the same-spin and opposite-spin parts, the two-parameter adjustment will only be a slight improvement over the one-parameter scaling scheme. Furthermore, optimizing coefficients of such linearly dependent variables constitutes a condition of overfitting, leading to a potential misbehavior of the adjusted parameters. Certainly, the appearance of a negative coefficient is indeed just such a problem. When $c_{U}^{\text {ss }}$ was set to be zero (limiting value within the physically meaningful range), the best fit was found with $c_{U}^{\mathrm{OS}}=1.54$. With these parameters, the RMS fit error was $0.18 \mathrm{eV}$. Again, this 
minute degradation $(<0.01 \mathrm{eV})$ in the RMS fit error is an evidence that the improvement upon using a two-parameter fit of SCS-CIS(D) does not have a physical origin, at least with respect to the data set we have employed.

\section{Performance Analysis}

A. Comparison between Scaling Methods. It will be interesting to directly compare the proposed scaling methods against the original CIS(D) method. Table 1 presents the transition energies of various electronic transitions from the training set from these methds. The excitation energies listed in the table are visually compared in Figure 6. One can clearly see that both SCS-CIS(D) and SOS-CIS(D) show good agreement with experiment for a wide range of transition energies $(2-12 \mathrm{eV})$. More direct comparison of the two methods can be made with Figure 7, where their errors within the training set are presented visually. The improvement is clear when it is compared with the performance of RI-CIS(D) in Figure 3. The mean absolute errors (MAEs) of SCSand SOS-CIS(D) are both $0.13 \mathrm{eV}$, while the MAE of RI-CIS(D) is $0.30 \mathrm{eV}$. (It is interesting to note that these numerical performances are comparable or better than TDDFT for the molecules in the test set. ${ }^{27}$ ) For RI-CIS(D), as explained in the previous section, there exist systematic overestimations for valence transitions and systematic underestimations for Rydberg transitions: the method tends to give larger transition energies than experiment as represented by its mean signed errors (MSEs) of $0.19 \mathrm{eV}$ in the subset of valence transitions and $-0.54 \mathrm{eV}$ in the subset of Rydberg transitions. This systematic error is directly corrected in the SCS- and SOS-CIS(D) approaches by adjustment of the empirical scaling factors and the damping parameter: the MSE of the 
proposed methods for valence and Rydberg transitions are only $0.02 \mathrm{eV}$ and $-0.08 \mathrm{eV}$, respectively.

Accordingly, it can be concluded that the benefits of the new methods over conventional CIS(D) are the correction of its systematic errors, and the improvement of the efficiency through the use of RI approximation. From the above observations of the errors of the two proposed methods, and from the fact that the optimal value of $c_{U}^{\mathrm{sS}}$ is found to be physically unimportant (in turn, it was set to be zero), we expect that the benefit of using SCS-CIS(D) approach over SOS-CIS(D) will be marginal. In the case of SOS-CIS(D), the efficiency improvement will be more dramatic for large molecules as it can be implemented with a fourth order scaling algorithm. The actual cost analysis will be discussed in a later part of this section.

Even though the reduced error is a desirable feature of SCS- and SOS-CIS(D) in comparison with the unscaled CIS(D) approach, this improvement is expected from the formulation of the two methods. However, the potential of describing various transitions in a balanced way will be an important feature in real applications of the proposed methods. Such applications outside of the training set will also be discussed later.

From Table 1, we can also compare our approach to producing a spin-component scaled CIS(D) method against the previous suggestion. ${ }^{27}$ This earlier approach ${ }^{27}$ scaled the indirect term using the ground state SCS-MP2 parameters, as we do, but did not scale the direct term (in terms of our eq 23, it is the special case where $c_{U}^{\mathrm{oS}}=1.00$ and $c_{U}^{\mathrm{sS}}=$ 1.00 together with $\lambda=1.00$ ). The results for this incompletely optimized form of SCSCIS(D) from Table 1 show systematic deviations that are similar to CIS(D) itself. The MSE and the MAE in the valence transitions for this scaling of only the indirect term of 
SCS-CIS(D) were reported to be both $0.22 \mathrm{eV},{ }^{27}$ showing that the systematic error associated with CIS(D) is still present. The MSE for Rydberg transitions is found to be $-0.59 \mathrm{eV}$, which is a similar tendency to CIS(D) itself. Overall, the MAE of this previous suggestion is $0.32 \mathrm{eV}$. The reduction of the MAE to $0.13 \mathrm{eV}$ in our fully optimized SCS-CIS(D) reflects elimination of the systematic component of the error when the direct term is scaled, and the value of optimizing the damping factor.

B. Application to a Charge Transfer Transition. As described in Introduction, the major motivation for a development of low cost CIS(D)-like methods is to attain a methodology that is applicable to large systems for which the more widely-used TDDFT approach fails. Therefore, it will be important to demonstrate that the present method is indeed reliable for such a system. One such example is the zincbacteriochlorinbacteriochlorin (ZnBC-BC) complex model (shown in Figure 8), previously examined by Dreuw and Head-Gordon. ${ }^{11}$

Figure 9 presents potential energy curves for the lowest charge transfer excited state from vertical excitation energies at various distances between the $\mathrm{ZnBC}$ and $\mathrm{BC}$ moieties. The same basis set $\left(6-31 G^{*}\right)$ and geometries reported in ref 11 have been adopted for this calculation. For comparison, results from SCS-CIS(D), SOS-CIS(D), RI-CIS(D), CIS, and TDDFT/B3LYP are shown in the figure. From the figure, it is apparent that TDDFT fails for charge transfer excitations as was already reported (significant underestimation of the energy and incorrect asymptotic behavior). ${ }^{11}$ In contrast, all other (wave-function based) methods give correct asymptotic behavior ( $1 / R)$. Apparently, CIS tends to overestimate the transition energies, while all correlation 
corrected CIS(D) variants give essentially the same results (within the uncertainly level of the methods). From these results, we can ascertain the appropriateness of the proposed methods in the description of charge transfer transitions.

Interestingly, when the four spin components (eq 23) of RI-CIS(D) were individually inspected, it was found that they have very weak dependence on the monomer separation $R$ in the inspected range (less than $0.02 \mathrm{eV}$ difference). This insensitivity is the reason for the agreement of all the CIS(D) variants and for the correct $1 / R$ behavior of CIS. Namely, electron correlation effects do not strongly influence the asymptotic $1 / R$ behavior, as they essentially serve as a constant shift in this region. In fact, this presents another justification of the hybrid scheme to obtain the correct excited state curve by combining both DFT and CIS results. ${ }^{8,11}$ However, the situation will change at short $R$. The error of CIS is due to the difference in the correlations on the ground and the excited electronic states. (Namely, if the correlations are the same on the two states, CIS will give the correct result.) When the monomers are in close contact, the electron correlation will strongly depend on the separation $R$, and it will be highly likely that the dependence on the excited state is considerably different from that on the ground state case. In such a region, the shape of the potential curve from CIS may be considerably different from the results obtained with CIS(D) methods.

\section{Comparison with Coupled-Cluster Results and Experiment. To obtain a}

further detailed benchmark of the proposed methods, it will be useful to compare their results with highly reliable (and highly computationally expensive) coupled cluster numbers. Table 2 presents the vertical excitation energies calculated from SCS-CIS(D) 
and SOS-CIS(D) for various small molecules together with the results from EOMCC $(2,3)^{43}$ or EOM-CCSDT-3. ${ }^{44,45}$ Overall, one can see that the scaled results are in good agreement with the coupled-cluster numbers. One important outlier from this trend is the symmetric $\left({ }^{1} \mathrm{~A}_{\mathrm{g}}\right)$ excited state of butadiene. It is well known that this state has a significant contribution from double-excitations, ${ }^{46}$ as is also exemplified by $39.4 \%$ doubles' contribution obtained with EOM-CC(2,3) amplitudes. When the doubles' contribution becomes large, any perturbative scheme that uses CIS state as its reference becomes unreliable. ${ }^{47}$ Clearly, this is a limitation of the present methods. When this outlier is excluded from the list, both SCS-CIS(D) and SOS-CIS(D) present an error level (mean absolute error of $0.16 \mathrm{eV}$ ) that is similar to the one previously obtained with the training set (i.e. comparing against experimental data for molecules in Table 1).

Similar agreement is also found when the two methods are compared against experimental results beyond the training set. Table 3 shows the scaled excitation energies in comparison with well-characterized experimental transition energies. ${ }^{48-50}$ Except for two outliers from $\mathrm{CH}_{2} \mathrm{O}\left(2^{1} \mathrm{~A}_{1}\right.$ and $\left.3^{1} \mathrm{~A}_{2}\right)$, both SCS-CIS(D) and SOS-CIS(D) show good agreements with experiment with MAE of $0.17 \mathrm{eV}$. Interestingly, these two outliers again can be well explained theoretically: both states mix strongly with other nearly degenerate states (the largest mixing angles obtained with the theta diagnostic ${ }^{47}$ are $16.4^{\circ}$ and $16.1^{\circ}$ for these states, whereas other states of $\mathrm{CH}_{2} \mathrm{O}$ in the table have mixing angles of $1^{\circ}-6^{\circ}$ ). This is another case where a perturbative treatment using a CIS reference state may fail. To obtain a proper description of such states, a quasi-degenerate perturbation correction approach ${ }^{14,29}$ is necessary. 
D. Timing. In this section, we shall address two main issues. First is the impact of the use of the auxiliary basis on timings for evaluation of CIS(D) and SCS-CIS(D), and second is the comparison between the SCS and SOS approaches, which scale differently with system size. The first issue can be addressed by the timings shown in Table 4. In the left-hand column are CIS(D) timings produced using semidirect methods based on exact evaluation of 4-center 2-electron integrals, as described previously. ${ }^{29}$ In the second column are SCS-CIS(D) timings computed using the auxiliary basis algorithms described and implemented in this work. It is evident that while the formal $5^{\text {th }}$ order scaling is identical in the two algorithms, the use of the auxiliary basis expansions provides a dramatic reduction in the value of the prefactor - which is reduced by between one and two orders of magnitude. This strongly supports the value of the auxiliary basis approach to CIS(D) and SCS-CIS(D) excitation energies.

Turning to the second issue, we recall from the results discussed in previous subsections that SCS-CIS(D) and SOS-CIS(D) are comparable in terms of the accuracy of the excitation energies obtained. Thus, use of SOS-CIS(D) will be potentially preferable based on the expectation of reduced computational cost from its fourth order scaling characteristics. However, because its prefactor will be larger than SCS-CIS(D) mainly due to the additional loop for the Laplace quadrature points, it will be important to explore the cross-over point between the two methods.

In fact, the cross-over point can be estimated from the nominal costs of the two methods. Because the leading costs of SCS- and SOS-CIS(D) are $2 O^{2} V^{2} X S$ and $2 O V X^{2} S T$ (where $O$ is the number of occupied orbitals, $V$ is the number of virtuals, $X$ is the number of auxiliary functions, $S$ is the number of states requested, and $T$ is the number of 
quadrature points), it is easy to see that the two methods will cross over when $O \sim T X / V$. Given that the number of quadrature points $(T=7)$ is fixed and the ratio $X / V$ is rather insensitive to the basis set quality (ranging between $2-4$ depending on the size of the basis), the cross-over point for any given system will mainly depend on the number of occupied orbitals $(O)$, or the size of the system, and apparently will be in the vicinity of 15 to 30 occupied orbitals.

The comparison of the actual processor times of the two methods for various molecules in Table 1 is presented in Figure 10 (and Table 4). As can be easily inferred from the above explanation, the ratio grows linearly with the number of occupied orbitals. Also, the two methods actually cross over at $O \sim 25$ with the aug-cc-pVTZ basis we have used. Therefore, we can conclude that SOS-CIS(D) will be faster for molecules above this size than SCS-CIS(D) or RI-CIS(D). Indeed, the calculations of ten excited states for ZnBC-BC complex (55 heavy atoms, $O=199$, and 918 basis functions with 6-31G* basis set) required $50 \mathrm{CPU}$ hours on a single $2.0 \mathrm{GHz}$ Opteron processor with SOS-CIS(D). (In comparison, SCS-CIS(D) required 140 CPU hours.) Therefore, we conclude that SOS-CIS(D) will be significantly easier to apply to systems with more than 100 heavy atoms in combination with reasonable basis sets than either CIS(D) or SCS-CIS(D).

\section{Concluding Remarks}

We have developed new scaled excited state methods by individually considering the different spin components of the correlation energies in conventional CIS(D) theory. These methods, SCS-CIS(D) and SOS-CIS(D), are the excited state counterparts of the

recently proposed ground state methods, SCS-MP2 ${ }^{23}$ and SOS-MP2. ${ }^{24}$ While the two 
methods present comparable reliability in reproducing the experimental excitation energies, SOS-CIS(D) offers the key advantage of reduced cost through the use of the Laplace transform to attain fourth order, rather than fifth order scaling with system size.

SOS-CIS(D) has a number of desirable aspects as a practical method for large molecular systems. First, with only one empirical excited-state scaling parameter, excitation energies of at least CIS(D) quality can be obtained. Indeed significant improvements are obtained for Rydberg excited states. One scaling parameter can be applied universally for many different organic molecules (system-independent) as demonstrated by a wide range of calcualtions. In addition, as a self-interaction-free wavefunction-based methodology, the theory is applicable to transitions with charge transfer characteristics without any problems associated with approximate exchangecorrelation functionals of density-based methods. Most importantly, the theory can be implemented with computational complexity that scales only with the fourth power of the system size.

As in the case of other theories, the present method will have limitations in certain cases. Because the method is based on CIS(D), it may be inappropriate for systems where CIS(D) itself fails. Important examples are the cases where the single reference picture is not a valid description of the ground state, ${ }^{51}$ or where there is a near-degeneracy in the excited states of a given system, ${ }^{29}$ or where the excited state has significant contributions from double excitations or higher. (In fact, such difficulties are generic problems of many of presently available $a b$ initio methods for excited states.) However, when the method is carefully applied, SOS-CIS(D) will be useful for many molecular systems. With its attractive features of reliability and efficiency described above, the 
method may constitute a promising technique for characterizing electronic transitions in large molecular systems.

There are also a number of interesting possible extensions based on the twin successes of the tests reported here, and the reduced scaling of the algorithm. CIS(D) itself is based on non-degenerate perturbation theory (diagonalize via CIS then perturb with correlation). The quasi-degenerate generalizations of CIS(D) ${ }^{29}$ could be usefully reformulated using the scaled opposite spin (SOS) approach to yield an iterative fourth order scaling method that would be resistant to quasi-degeneracies. In a similar vein, the quasi-degenerate CC2 method could readily be recast to yield a fourth order scaling SOSCC2 approach that would be applicable to larger systems. These cases are particularly interesting because based on our treatment of the SOS-MP2 gradient, ${ }^{42}$ we suspect that they can be recast to avoid storage of fourth order amplitudes. ${ }^{22}$ We hope to report on this development in due course.

Acknowledgement. This work was supported by the Director, Office of Science, Office of Basic Energy Sciences, of the U.S. Department of Energy under Contract No. DE-AC02-05CH11231, and supercomputer time from NERSC. 


\section{References and Notes}

(1) Sekino, H.; Bartlett, R. J. Int. J. Quantum Chem. Symp. 1984, 18, 225.

(2) Emrich, K. Nucl. Phys. A 1981, 351, 392.

(3) Monkhorst, H. J. Int. J. Quantum Chem. Symp. 1977, 11, 421.

(4) Mukherjee, D.; Mukherjee, P. Chem. Phys. 1979, 39, 325.

(5) Koch, H.; Jørgensen, P. J. Chem. Phys. 1990, 93, 3333.

(6) Bartlett, R. J. Part 1. In Modern Electronic Structure Theory; Yarkony, D. R., Ed.; World Scientific: Singapore, 1995.

(7) Roos, B. O.; Andersson, K.; Fülscher, M. Chem. Phys. Lett. 1992, 192, 5.

(8) Dreuw, A.; Head-Gordon, M. Chem. Rev. 2005, 105, 4009.

(9) Gross, E. K. U.; Kohn, W. Phys. Rev. Lett. 1985, 55, 2850.

(10) Runge, E.; Gross, E. K. U. Phys. Rev. Lett. 1984, 52, 997.

(11) Dreuw, A.; Head-Gordon, M. J. Am. Chem. Soc. 2004, 126, 4007.

(12) Johnson, B. G.; Gonzales, C. A.; Gill, P. M. W.; Pople, J. A. Chem. Phys. Lett. 1994, 221, 100.

(13) Head-Gordon, M.; Rico, R. J.; Oumi, M.; Lee, T. J. Chem. Phys. Lett. 1994, 219, 21.

(14) Christiansen, O.; Koch, H.; Jørgensen, P. Chem. Phys. Lett. 1995, 243, 409.

(15) Frisch, M. J.; Head-Gordon, M.; Pople, J. A. Chem. Phys. Lett. 1990, 166, 281.

(16) Frisch, M. J.; Head-Gordon, M.; Pople, J. A. Chem. Phys. Lett. 1990, 166, 275.

(17) Vahtras, O.; Almlöf, J.; Feyereisen, M. W. Chem. Phys. Lett. 1993, 213, 514.

(18) Feyereisen, M.; Fitzgerald, G.; Komornicki, A. Chem. Phys. Lett. 1993, 208, 359. 
(19) Werner, H.-J.; Manby, F. R.; Knowles, P. J. J. Chem. Phys. 2003, 118, 8149.

(20) Whitten, J. L. J. Chem. Phys. 1973, 58, 4496.

(21) Hättig, C.; Hald, K. Phys. Chem. Chem. Phys. 2002, 4, 2111.

(22) Hättig, C.; Weigend, F. J. Chem. Phys. 2000, 113, 5154.

(23) Grimme, S. J. Chem. Phys. 2003, 118, 9095.

(24) Jung, Y.; Lochan, R. C.; Dutoi, A. D.; Head-Gordon, M. J. Chem. Phys. 2004, 121, 9793.

(25) Almlöf, J. Chem. Phys. Lett. 1991, 181, 319.

(26) Häser, M.; Almlöf, J. J. Chem. Phys. 1992, 96, 489.

(27) Grimme, S.; Izgorodina, E. I. Chem. Phys. 2004, 305, 223.

(28) Foresman, J. B.; Head-Gordon, M.; Pople, J. A.; Frisch, M. J. J. Phys. Chem. 1992, 96, 135.

(29) Head-Gordon, M.; Oumi, M.; Maurice, D. Mol. Phys. 1999, 96, 593.

(30) Koch, H.; Christiansen, O.; Jørgensen, P. J. Chem. Phys. 1990, 93, 3333.

(31) DiStasio, R. A.; Steele, R. P.; Rhee, Y. M.; Shao, Y.; Head-Gordon, M. J. Comput. Chem. 2007, 28, 839.

(32) Weigend, F.; Häser, M. Theo. Chem. Acc. 1997, 97, 331.

(33) Ayala, P. Y.; Scuseria, G. E. J. Chem. Phys. 1999, 110, 3660.

(34) Kendall, R. A.; Dunning, T. H.; Harrison, R. J. J. Chem. Phys. 1992, 96, 6796.

(35) Weigend, F.; Köhn, A.; Hättig, C. J. Chem. Phys. 2002, 116, 3175.

(36) Herzberg, G. Electronic spectra and electronic structure of polyatomic molecules; Van Nostrand: New York, 1966. 
(37) Winter, N. W.; Goddard, W. A.; Bobrowicz, F. W. J. Chem. Phys. 1975, 62, 4325.

(38) Bartlett, R. J.; Del Bene, J. E.; Perera, S. A.; Mattie, R. P. J. Mol. Struct.: THEOCHEM 1997, 400, 157.

(39) Shao, Y.; Molnar, L. F.; Jung, Y.; Kussmann, J.; Ochsenfeld, C.; Brown, S. T.; Gilbert, A. T. B.; Slipchenko, L. V.; Levchenko, S. V.; O’Neill, D. P.; Distasio, R. A.; Lochan, R. C.; Wang, T.; Beran, G. J. O.; Besley, N. A.; Herbert, J. M.; Lin, C. Y.; van Voorhis, T.; Chien, S. H.; Sodt, A.; Steele, R. P.; Rassolov, V. A.; Maslen, P. E.; Korambath, P. P.; Adamson, R. D.; Austin, B.; Baker, J.; Byrd, E. F. C.; Dachsel, H.; Doerksen, R. J.; Dreuw, A.; Dunietz, B. D.; Dutoi, A. D.; Furlani, T. R.; Gwaltney, S. R.; Heyden, A.; Hirata, S.; Hsu, C.-P.; Kedziora, G.; Khalliulin, R. Z.; Klunzinger, P.; Lee, A. M.; Lee, M. S.; Liang, W.; Lotan, I.; Nair, N.; Peters, B.; Proynov, E. I.; Pieniazek, P. A.; Rhee, Y. M.; Ritchie, J.; Rosta, E.; Sherrill, C. D.; Simmonett, A. C.; Subotnik, J. E.; Woodcock III, H. L.; Zhang, W.; Bell, A. T.; Chakraborty, A. K.; Chipman, D. M.; Keil, F. J.; Warshel, A.; Hehre, W. J.; Schaefer III, H. F.; Kong, J.; Krylov, A. I.; Gill, P. M. W.; Head-Gordon, M. Phys. Chem. Chem. Phys. 2006, 8, 3172.

(40) Wilson, A. K.; Almlöf, J. Theo. Chim. Acta 1997, 95, 49.

(41) Kobayashi, M.; Nakai, H. Chem. Phys. Lett. 2006, 420, 250.

(42) Lochan, R. C.; Shao, Y.; Head-Gordon, M. J. Chem. Theory Comput., in press.

(43) Hirata, S.; Nooijen, M.; Bartlett, R. J. Chem. Phys. Lett. 2000, 326, 255.

(44) Watts, J. D.; Bartlett, R. J. Chem. Phys. Lett. 1996, 258, 581.

(45) Bomble, Y. J.; Sattelmeyer, K. W.; Stanton, J. F.; Gauss, J. J. Chem. Phys. 2004, $121,5236$. 
(46) Hsu, C.-P.; Hirata, S.; Head-Gordon, M. J. Phys. Chem. A 2001, 105, 451.

(47) Oumi, M.; Maurice, D.; Lee, T. J.; Head-Gordon, M. Chem. Phys. Lett. 1997, 279, 151.

(48) Hadad, C. M.; Foresman, J. B.; Wiberg, K. B. J. Phys. Chem. 1993, 97, 4293.

(49) Wiberg, K. B.; Hadad, C. M.; Foresman, J. B.; Chupka, W. A. J. Phys. Chem. 1992, 96, 10756.

(50) Wiberg, K. B.; Hadad, C. M.; Ellison, B.; Foresman, J. B. J. Phys. Chem. 1993, $97,13586$.

(51) Helgaker, T.; Jørgensen, P.; Olsen, J. Molecular Electronic-Structure Theory; Wiley: New York, 2000. 


\section{Tables}

TABLE 1: List of electronic transitions adopted in the parameter optimizations. Transition energies with various methods are also presented in comparison with experimental values. Energies are in $\mathrm{eV}$ units.

\begin{tabular}{|c|c|c|c|c|c|c|c|c|c|}
\hline & Molecule & No. & Symmetry & CIS & $\begin{array}{c}\text { SCS- } \\
\text { CIS(D) }\end{array}$ & $\begin{array}{c}\text { SOS- } \\
\text { CIS(D) }\end{array}$ & $\begin{array}{c}\text { RI- } \\
\text { CIS(D) }\end{array}$ & $\begin{array}{c}\text { SCS- } \\
\text { CIS(D) }^{\prime a}\end{array}$ & Exp. ${ }^{b}$ \\
\hline \multirow[t]{20}{*}{$\pi \rightarrow \pi^{*}$} & Hexatriene & 1 & ${ }^{1} \mathrm{~B}_{\mathrm{u}}$ & 4.67 & 4.83 & 4.87 & 4.85 & 4.93 & 4.93 \\
\hline & \multirow[t]{3}{*}{ Benzene } & 2 & ${ }^{1} \mathrm{~B}_{1 \mathrm{u}}$ & 5.99 & 6.07 & 6.03 & 6.33 & 6.35 & 6.03 \\
\hline & & 3 & ${ }^{1} B_{2 u}$ & 5.84 & 4.77 & 4.75 & 5.10 & 4.91 & 4.72 \\
\hline & & 4 & ${ }^{1} \mathrm{E}_{1 \mathrm{u}}$ & 7.55 & 7.34 & 7.31 & 7.18 & 7.22 & 6.87 \\
\hline & Phenol & 5 & ${ }^{1} \mathrm{~A}^{\prime}$ & 5.57 & 4.51 & 4.49 & 4.79 & 4.57 & 4.51 \\
\hline & Benzaldehyde & 6 & ${ }^{1} \mathrm{~A}^{\prime}$ & 5.24 & 5.12 & 5.09 & 5.33 & 5.46 & 5.12 \\
\hline & \multirow[t]{2}{*}{ Styrene } & 7 & ${ }^{1} \mathrm{~A}^{\prime}$ & 4.57 & 4.81 & 4.79 & 4.94 & 4.92 & 4.88 \\
\hline & & 8 & ${ }^{1} \mathrm{~A}^{\prime}$ & 5.53 & 4.54 & 4.52 & 4.79 & 4.68 & 4.31 \\
\hline & Octatetraene & 9 & ${ }^{1} \mathrm{~B}_{\mathrm{u}}$ & 4.17 & 4.24 & 4.28 & 4.25 & 4.38 & 4.41 \\
\hline & \multirow[t]{2}{*}{ Naphthalene } & 10 & ${ }^{1} \mathrm{~B}_{2 \mathrm{u}}$ & 4.46 & 4.46 & 4.44 & 4.61 & 4.60 & 4.45 \\
\hline & & 11 & ${ }^{1} B_{3 u}$ & 4.91 & 3.99 & 3.96 & 4.28 & 4.10 & 3.96 \\
\hline & Azulene & 12 & ${ }^{1} \mathrm{~B}_{1}$ & 2.33 & 1.56 & 1.52 & 1.97 & 1.83 & 1.77 \\
\hline & \multirow[t]{2}{*}{ Indole } & 13 & ${ }^{1} \mathrm{~A}^{\prime}$ & 4.99 & 4.86 & 4.83 & 4.99 & 5.08 & 4.54 \\
\hline & & 14 & ${ }^{1} \mathrm{~A}^{\prime}$ & 5.33 & 4.46 & 4.43 & 4.68 & 4.77 & 4.37 \\
\hline & $p$-Diethynylbenzene & 15 & ${ }^{1} \mathrm{~B}_{2 \mathrm{u}}$ & 5.46 & 4.34 & 4.32 & 4.59 & 4.46 & 4.25 \\
\hline & Biphenylene & 16 & ${ }^{1} \mathrm{~B}_{3 \mathrm{u}}$ & 4.39 & 3.56 & 3.53 & 3.74 & 3.74 & 3.55 \\
\hline & trans-Stilbene & 17 & ${ }^{1} \mathrm{~B}_{\mathrm{u}}$ & 3.77 & 3.91 & 3.89 & 4.03 & 4.18 & 4.00 \\
\hline & Anthracene & 18 & ${ }^{1} \mathrm{~B}_{2 \mathrm{u}}$ & 3.52 & 3.34 & 3.32 & 3.42 & 3.55 & 3.43 \\
\hline & \multirow{2}{*}{ Pyrene } & 19 & ${ }^{1} \mathrm{~B}_{2 \mathrm{u}}$ & 4.12 & 3.87 & 3.82 & 3.98 & 4.03 & 3.81 \\
\hline & & 20 & ${ }^{1} \mathrm{~B}_{3 \mathrm{u}}$ & 4.33 & 3.33 & 3.28 & 3.56 & 3.47 & 3.44 \\
\hline \multirow[t]{12}{*}{$n \rightarrow \pi^{*}$} & Acetone & 21 & ${ }^{1} \mathrm{~A}_{2}$ & 4.95 & 3.89 & 3.94 & 4.10 & 3.85 & 3.76 \\
\hline & \multirow[t]{2}{*}{ Thioacetone } & 22 & ${ }^{1} \mathrm{~A}_{2}$ & 3.14 & 2.15 & 2.20 & 2.42 & 2.37 & 2.33 \\
\hline & & 23 & ${ }^{3} \mathrm{~A}_{2}$ & 2.54 & 2.02 & 2.06 & 2.20 & 2.24 & 2.14 \\
\hline & \multirow[t]{2}{*}{ Acrolein } & 24 & ${ }^{1} \mathrm{~A}^{\prime \prime}$ & 4.47 & 3.35 & 3.42 & 3.51 & 3.66 & 3.21 \\
\hline & & 25 & ${ }^{3} \mathrm{~A}^{\prime \prime}$ & 3.70 & 3.15 & 3.22 & 3.25 & 3.31 & 3.01 \\
\hline & \multirow[t]{2}{*}{ 2-Cyclopenten-1-one } & 26 & ${ }^{1} \mathrm{~A}^{\prime \prime}$ & 4.81 & 3.54 & 3.59 & 3.66 & 3.73 & 3.36 \\
\hline & & 27 & ${ }^{3} \mathrm{~A}^{\prime \prime}$ & 4.20 & 3.38 & 3.42 & 3.46 & 3.56 & 3.22 \\
\hline & s-Tetrazine & 28 & ${ }^{1} \mathrm{~B}_{1 \mathrm{u}}$ & 3.15 & 2.19 & 2.20 & 2.39 & 2.72 & 2.25 \\
\hline & \multirow[t]{2}{*}{ Benzaldehyde } & 29 & ${ }^{1} \mathrm{~A}^{\prime \prime}$ & 4.56 & 3.37 & 3.42 & 3.51 & 3.65 & 3.34 \\
\hline & & 30 & ${ }^{3} \mathrm{~A}^{\prime \prime}$ & 3.83 & 3.18 & 3.23 & 3.27 & 3.37 & 3.12 \\
\hline & DMABN & 31 & ${ }^{1} \mathrm{~A}_{2}$ & 5.26 & 4.01 & 3.98 & 4.20 & 4.32 & 3.95 \\
\hline & trans-Azobenzene & 32 & ${ }^{1} \mathrm{~A}_{2}$ & 2.33 & 2.21 & 2.24 & 2.49 & 2.85 & 2.60 \\
\hline Rydberg & \multirow[t]{7}{*}{ Water } & 33 & ${ }^{1} \mathrm{~B}_{1}$ & 8.64 & 7.20 & 7.24 & 6.98 & 6.92 & 7.49 \\
\hline \multirow[t]{10}{*}{ States } & & 34 & ${ }^{1} \mathrm{~A}_{2}$ & 10.32 & 9.10 & 9.13 & 8.74 & 8.67 & 9.20 \\
\hline & & 35 & ${ }^{1} \mathrm{~A}_{1}$ & 10.91 & 9.67 & 9.71 & 9.30 & 9.25 & 9.73 \\
\hline & & 36 & ${ }^{1} \mathrm{~B}_{1}$ & 11.30 & 9.78 & 9.81 & 9.24 & 9.17 & 10.00 \\
\hline & & 37 & ${ }^{1} \mathrm{~A}_{1}$ & 11.57 & 9.92 & 9.95 & 9.30 & 9.23 & 10.17 \\
\hline & & 38 & ${ }^{1} \mathrm{~B}_{2}$ & 12.65 & 11.60 & 11.63 & 11.07 & 11.02 & 11.50 \\
\hline & & 39 & ${ }^{1} \mathrm{~A}_{1}$ & 13.47 & 12.25 & 12.28 & 11.56 & 11.50 & 12.10 \\
\hline & \multirow[t]{4}{*}{ Ammonia } & 40 & ${ }^{1} \mathrm{~A}_{2} "$ & 7.34 & 6.28 & 6.32 & 6.13 & 6.09 & 6.38 \\
\hline & & 41 & ${ }^{1} E^{\prime \prime}$ & 8.78 & 7.85 & 7.88 & 7.55 & 7.51 & 7.91 \\
\hline & & 42 & ${ }^{1} \mathrm{~A}_{1}^{\prime}$ & 9.31 & 8.25 & 8.28 & 7.89 & 7.85 & 8.26 \\
\hline & & 43 & ${ }^{1} \mathrm{~A}_{2}{ }^{\prime \prime}$ & 9.88 & 8.76 & 8.79 & 8.31 & 8.27 & 9.25 \\
\hline Mean sigr & error & & & 0.75 & -0.01 & 0.00 & 0.01 & 0.02 & \\
\hline Mean abs & te error & & & 0.81 & 0.13 & 0.13 & 0.30 & 0.32 & \\
\hline
\end{tabular}

${ }^{a}$ With spin component scaling only on the indirect term. Excitation energies of valence transitions are from ref 27.

${ }^{\mathrm{b}}$ Experimental data are taken from the compilations of ref 27 (organic molecules), ref 37 (water), and ref 38 (ammonia). 
TABLE 2: List of vertical excitation energies of molecules with varying sizes. SCSand SOS-CIS(D) results are compared with equation-of-the-motion coupled-cluster results. Transition energies are in $\mathrm{eV}$ units.

\begin{tabular}{|c|c|c|c|c|c|}
\hline Molecule & $\begin{array}{c}\text { Excited state } \\
\text { symmetry }\end{array}$ & $\begin{array}{c}\text { Transition } \\
\text { character }\end{array}$ & $\begin{array}{c}\text { SCS- } \\
\text { CIS(D) }\end{array}$ & $\begin{array}{c}\text { SOS- } \\
\text { CIS(D) }\end{array}$ & $\begin{array}{c}\text { EOM- } \\
\text { CC } \\
\end{array}$ \\
\hline \multirow[t]{3}{*}{$\mathrm{CO}^{\mathrm{a}}$} & ${ }^{1} \Delta$ & $\pi \rightarrow \pi^{*}$ & 10.19 & 10.21 & $10.13^{\mathrm{b}}$ \\
\hline & ${ }^{1} \Sigma^{-}$ & $\pi \rightarrow \pi^{*}$ & 10.02 & 10.05 & $10.03^{b}$ \\
\hline & ${ }^{1} \Pi$ & $\sigma \rightarrow \pi^{*}$ & 8.81 & 8.87 & $8.70^{\mathrm{b}}$ \\
\hline $\mathrm{CH}_{2} \mathrm{O}^{\mathrm{a}}$ & ${ }^{1} \mathrm{~A}_{2}$ & $n \rightarrow \pi^{*}$ & 3.85 & 3.92 & $4.09^{b}$ \\
\hline $\mathrm{C}_{2} \mathrm{H}_{4}{ }^{\mathrm{a}}$ & ${ }^{1} \mathrm{~B}_{3 \mathrm{u}}$ & $\pi \rightarrow \pi^{*}$ & 8.08 & 8.11 & $8.20^{\mathrm{b}}$ \\
\hline \multirow[t]{2}{*}{$\mathrm{CH}_{3} \mathrm{CHO}^{\mathrm{a}}$} & ${ }^{1} A^{\prime \prime}$ & $n \rightarrow \pi^{*}$ & 4.16 & 4.21 & $4.53^{b}$ \\
\hline & ${ }^{1} \mathrm{~A}^{\prime}$ & $\pi \rightarrow \pi^{*}$ & 6.93 & 6.96 & $7.15^{b}$ \\
\hline \multirow[t]{2}{*}{ trans-Butadiene $^{\mathrm{a}}$} & ${ }^{1} \mathrm{~B}_{\mathrm{u}}$ & $\pi \rightarrow \pi^{*}$ & 6.46 & 6.48 & $6.72^{b}$ \\
\hline & ${ }^{1} \mathrm{~A}_{\mathrm{g}}$ & $\pi \rightarrow \pi^{*}$ & 7.73 & 7.73 & $6.84^{\mathrm{b}}$ \\
\hline Cyclopentadiene $^{\mathrm{c}}$ & ${ }^{1} \mathrm{~B}_{1}$ & $\pi \rightarrow \pi^{*}$ & 5.93 & 5.94 & $5.90^{\mathrm{d}}$ \\
\hline
\end{tabular}

${ }^{a}$ Geometries were optimized at MP2/6-311(+,+)G(d,p) level. For $\mathrm{CO}, \mathrm{CH}_{2} \mathrm{O}, \mathrm{C}_{2} \mathrm{H}_{4}$, and $\mathrm{CH}_{3} \mathrm{CHO}$, the excitation energies were calculated with the same 6-311(+,+)G(d,p) basis. For RI-approximated integrals in these molecules, an auxiliary basis set corresponding to aug-cc-pVTZ was used. For trans-butadiene, $6-31+G(d)$ basis was used in conjunction with the auxiliary basis of aug-cc-pVDZ.

${ }^{\mathrm{b}} \mathrm{EOM}-\mathrm{CC}(2,3)$ results.

${ }^{\mathrm{c}}$ Geometry is from ref 45 . The same basis set in the reference (cc-pVDZ) was also adopted.

${ }^{\mathrm{d}}$ EOM-CCSDT-3 result taken from ref 45. 
TABLE 3: Comparison of theoretical and experimental excitation energies for valence $(V)$ and Rydberg $(R)$ transitions of various molecules. Transition energies are in $\mathrm{eV}$ units.

\begin{tabular}{|c|c|c|c|c|c|c|}
\hline Molecule $^{\mathrm{a}}$ & $\begin{array}{l}\text { Excited state } \\
\text { symmetry }\end{array}$ & $\begin{array}{l}\text { Transition } \\
\text { Character }\end{array}$ & $\mathrm{RI}^{-C I S(D)}{ }^{\mathrm{b}}$ & SCS-CIS(D) & SOS-CIS(D) & Exp. $^{\mathrm{a}}$ \\
\hline \multirow[t]{7}{*}{$\mathrm{CH}_{2} \mathrm{O}$} & ${ }^{1} \mathrm{~A}_{2}$ & $\mathrm{~V}$ & 4.03 & 3.85 & 3.92 & 4.07 \\
\hline & ${ }^{1} \mathrm{~B}_{2}$ & $\mathrm{R}$ & 6.44 & 7.06 & 7.11 & 7.11 \\
\hline & ${ }^{1} \mathrm{~B}_{2}$ & $\mathrm{R}$ & 7.27 & 7.91 & 7.95 & 7.97 \\
\hline & ${ }^{1} \mathrm{~A}_{1}$ & $\mathrm{R}$ & 9.01 & 9.27 & 9.31 & 8.14 \\
\hline & ${ }^{1} \mathrm{~A}_{2}$ & $\mathrm{R}$ & 7.49 & 8.21 & 8.26 & 8.37 \\
\hline & ${ }^{1} \mathrm{~B}_{2}$ & $\mathrm{R}$ & 8.21 & 9.06 & 9.10 & 8.88 \\
\hline & ${ }^{1} \mathrm{~A}_{2}$ & $\mathrm{~V}$ & 9.44 & 10.03 & 10.07 & 9.22 \\
\hline \multirow[t]{10}{*}{$\mathrm{C}_{2} \mathrm{H}_{4}$} & ${ }^{1} \mathrm{~B}_{3 \mathrm{u}}$ & $\mathrm{R}$ & 7.19 & 7.38 & 7.38 & 7.11 \\
\hline & ${ }^{1} B_{1 g}$ & $\mathrm{R}$ & 7.82 & 8.04 & 8.04 & 7.80 \\
\hline & ${ }^{1} \mathrm{~B}_{1 \mathrm{u}}$ & V & 8.02 & 8.05 & 8.08 & 7.60 \\
\hline & ${ }^{1} \mathrm{~B}_{2 \mathrm{~g}}$ & $\mathrm{R}$ & 7.84 & 8.09 & 8.09 & 8.01 \\
\hline & ${ }^{1} \mathrm{~A}_{\mathrm{g}}$ & $\mathrm{R}$ & 8.16 & 8.38 & 8.38 & 8.29 \\
\hline & ${ }^{1} \mathrm{~B}_{3 \mathrm{u}}^{\mathrm{s}}$ & $\mathrm{R}$ & 8.66 & 8.95 & 8.94 & 8.62 \\
\hline & ${ }^{1} B_{1 g}$ & $\mathrm{R}$ & 9.10 & 9.41 & 9.41 & 9.34 \\
\hline & ${ }^{1} \mathrm{~B}_{1 \mathrm{u}}$ & $\mathrm{R}$ & 9.15 & 9.43 & 9.43 & 9.33 \\
\hline & ${ }^{1} \mathrm{~B}_{3 \mathrm{u}}$ & $\mathrm{R}$ & 9.19 & 9.43 & 9.43 & 8.90 \\
\hline & ${ }^{1} \mathrm{~B}_{1 \mathrm{~g}}$ & $\mathrm{~V}$ & 8.81 & 8.98 & 9.06 & 9.20 \\
\hline \multirow[t]{6}{*}{$\mathrm{CH}_{3} \mathrm{CHO}$} & ${ }^{1} A^{\prime \prime}$ & $\mathrm{V}$ & 4.33 & 4.15 & 4.20 & 4.28 \\
\hline & ${ }^{1} \mathrm{~A}^{\prime}$ & $\mathrm{R}$ & 6.15 & 6.83 & 6.86 & 6.82 \\
\hline & ${ }^{1} A^{\prime}$ & $\mathrm{R}$ & 6.84 & 7.60 & 7.63 & 7.46 \\
\hline & ${ }^{1} \mathrm{~A}^{\prime}$ & $\mathrm{R}$ & 7.37 & 8.00 & 8.03 & 7.75 \\
\hline & ${ }^{1} \mathrm{~A}^{\prime}$ & $\mathrm{R}$ & 7.58 & 8.48 & 8.50 & 8.43 \\
\hline & ${ }^{1} \mathrm{~A}^{\prime}$ & $\mathrm{R}$ & 7.90 & 8.74 & 8.77 & 8.69 \\
\hline \multirow[t]{10}{*}{ trans $-\mathrm{C}_{4} \mathrm{H}_{6}$} & ${ }^{1} \mathrm{~B}_{\mathrm{g}}$ & $\mathrm{R}$ & 6.14 & 6.40 & 6.39 & 6.22 \\
\hline & ${ }^{1} \mathrm{~B}_{\mathrm{u}}$ & $\mathrm{V}$ & 6.26 & 6.35 & 6.37 & 5.91 \\
\hline & ${ }^{1} \mathrm{~A}_{\mathrm{u}}$ & $\mathrm{R}$ & 6.58 & 6.89 & 6.88 & 6.66 \\
\hline & ${ }^{1} \mathrm{~B}_{\mathrm{u}}$ & $\mathrm{R}$ & 7.04 & 7.28 & 7.28 & 7.07 \\
\hline & ${ }^{1} \mathrm{~A}_{\mathrm{g}}$ & $\mathrm{R}$ & 7.19 & 7.48 & 7.47 & 7.40 \\
\hline & ${ }^{1} \mathrm{~B}_{\mathrm{g}}$ & $\mathrm{R}$ & 7.20 & 7.55 & 7.54 & 7.36 \\
\hline & ${ }^{1} \mathrm{~B}_{\mathrm{g}}$ & $\mathrm{R}$ & 7.26 & 7.59 & 7.58 & 7.62 \\
\hline & ${ }^{1} \mathrm{~B}_{\mathrm{g}}^{\mathrm{g}}$ & $\mathrm{R}$ & 7.39 & 7.73 & 7.71 & 7.72 \\
\hline & ${ }^{1} \mathrm{~A}_{\mathrm{u}}$ & $\mathrm{R}$ & 7.68 & 8.06 & 8.05 & 8.18 \\
\hline & ${ }^{1} \mathrm{~A}_{\mathrm{u}}$ & $\mathrm{R}$ & 7.89 & 8.29 & 8.28 & 8.00 \\
\hline
\end{tabular}

\footnotetext{
${ }^{a}$ Experimental numbers and R/V assignments are from compilations of ref $48\left(\mathrm{CH}_{2} \mathrm{O}\right.$ and $\left.\mathrm{CH}_{3} \mathrm{CHO}\right)$, ref 49 $\left(\mathrm{C}_{2} \mathrm{H}_{4}\right)$, and ref $50\left(\mathrm{C}_{4} \mathrm{H}_{6}\right)$. Geometries were optimized at an MP2/6-31G(d) level.

${ }^{\mathrm{b}}$ Excitation energies were calculated with the 6-311(2+,2+)G(d,p) basis. For RI-approximated integrals, an auxiliary basis set corresponding to aug-cc-pVTZ basis was adopted.
} 
TABLE 4: CPU times for calculating 10 excited state energies of various molecules. ${ }^{\text {a }}$

\begin{tabular}{|c|c|c|c|c|}
\hline \multirow{2}{*}{ Molecule } & \multirow{2}{*}{$\begin{array}{l}\text { No. of } \\
\text { basis }\end{array}$} & \multicolumn{3}{|c|}{ CPU time (min) } \\
\hline & & $\mathrm{CIS}(\mathrm{D})^{\mathrm{b}}$ & SCS-CIS(D) & SOS-CIS(D) \\
\hline Acrolein $\left(\mathrm{C}_{3} \mathrm{H}_{4} \mathrm{O}\right)$ & $276^{c}$ & 65 & 3 & 4 \\
\hline Thioacetone $\left(\mathrm{C}_{3} \mathrm{H}_{4} \mathrm{~S}\right)$ & $326^{\mathrm{c}}$ & 225 & 7 & 8 \\
\hline Hexatriene $\left(\mathrm{C}_{6} \mathrm{H}_{8}\right)$ & $460^{c}$ & 942 & 23 & 25 \\
\hline Styrene $\left(\mathrm{C}_{8} \mathrm{H}_{8}\right)$ & $552^{\mathrm{C}}$ & 4944 & 57 & 55 \\
\hline Azulene $\left(\mathrm{C}_{10} \mathrm{H}_{8}\right)$ & $644^{\mathrm{C}}$ & - & 126 & 109 \\
\hline Anthracene $\left(\mathrm{C}_{14} \mathrm{H}_{10}\right)$ & $874^{\mathrm{C}}$ & - & 504 & 352 \\
\hline Pyrene $\left(\mathrm{C}_{16} \mathrm{H}_{10}\right)$ & $966^{\mathrm{c}}$ & - & 809 & 528 \\
\hline $\mathrm{ZnBC}-\mathrm{BC}\left(\mathrm{C}_{46} \mathrm{H}_{36} \mathrm{~N}_{8} \mathrm{Zn}\right)$ & $918^{\mathrm{d}}$ & - & 8372 & 3010 \\
\hline
\end{tabular}




\section{Figures}

Loop over $i$-batch (batch size: $B$ )

Disk IO cost $\quad$ CPU cost

5

\section{6}

7

8

9

10

11

12

13

14

15

16

17

18

19

20
Read $B_{a i}^{P}$ block

Read $D_{a i}^{P}$ block

Loop over $j$-batch (batch size: $B$ )

$$
\text { Read } B_{b j}^{P} \text { block }
$$

Read $D_{b j}^{P}$ block

Loop over $i(i \in i$-batch)

Loop over $j(j \in j$-batch)
OVX

OVXS

$O^{2} V X / B$

$O^{2} V X S / B$

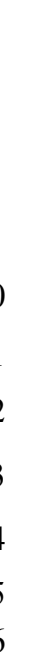

\begin{tabular}{|c|c|c|}
\hline$(i a \mid j b)=\sum_{P} B_{a i}^{P} B_{b j}^{P}$ & & $O^{2} V^{2} X$ \\
\hline Write $(i a \mid j b)$ & $O^{2} V^{2}$ & \\
\hline$a_{i j}^{a b}=[(i a \mid j b)-(i b \mid j a)] / \Delta_{i j}^{a b}$ & & $O^{2} V^{2}$ \\
\hline Write $a_{i j}^{a b}$ & $O^{2} V^{2}$ & \\
\hline$R_{a b}-=\sum_{c}(i b \mid j c) a_{i j}^{a c}$ & & $O^{2} V^{3}$ \\
\hline$(B D)_{i j}^{a b}=\sum_{P} B_{a i}^{P} D_{b j}^{P}$ & & $O^{2} V^{2} X S$ \\
\hline$(D B)_{i j}^{a b}=\sum_{P} D_{a i}^{P} B_{b j}^{P}$ & & $O^{2} V^{2} X S$ \\
\hline$u_{i j}^{a b}=(D B)_{i j}^{a b}-(D B)_{i j}^{b a}+(B D)_{i j}^{a b}-(B D)_{i j}^{b a}$ & & \\
\hline$\omega^{\operatorname{CIS}(\mathrm{D})}+=\frac{1}{4}\left(u_{i j}^{a b}\right)^{2} /\left(\Delta_{i j}^{a b}-\omega\right)$ & & \\
\hline$y_{i}^{a}+=\sum_{b}(i j \| a b) b_{j}^{b} \quad(i \in i$-batch, $a \in$ VIRT $)$ & & \\
\hline Re-order $(i a \mid j b)$ on disk: $(b, j, a, i) \leftarrow(b, a, j, i)$ & $O^{2} V^{2}$ & \\
\hline Re-order $a_{i j}^{a b}$ on disk $\quad:(b, j, a, i) \leftarrow(b, a, j, i)$ & $O^{2} V^{2}$ & \\
\hline Loop over $i(i \in i$-batch $)$ & & \\
\hline Loop over $a(a \in$ VIRT $)$ & & \\
\hline $\operatorname{Read}(i a \mid j b)$ & $O^{2} V^{2}$ & \\
\hline Read $a_{i j}^{a b}$ & $O^{2} V^{2}$ & \\
\hline$R_{j k}-=\sum_{b}(i a \mid j b) a_{i k}^{a b}$ & & $O^{3} V^{2}$ \\
\hline$w_{k}^{c}+=y_{i}^{a} a_{i k}^{a c} \quad(k \in \mathrm{OCC}, c \in \mathrm{VIRT})$ & & \\
\hline
\end{tabular}

Figure 1. Algorithm for RI-CIS(D) theory and its cost. SCS-CIS(D) can be implemented by separately evaluating same-spin and opposite-spin components at Lines 9, 12, 19, and 20. Loops for the excited states (for Lines $10-14$ and Line 20) are omitted for visual clarity. For CPU cost, only fifth order scaling routines are noted. 
Loop over $t$ ( $t \in$ quadrature points, size: $T$ )

Disk IO cost CPU cost

Loop over $s$ ( $s \in$ excited states, size: $S$ ) Loop over $i$ ( $i \in \mathrm{OCC})$

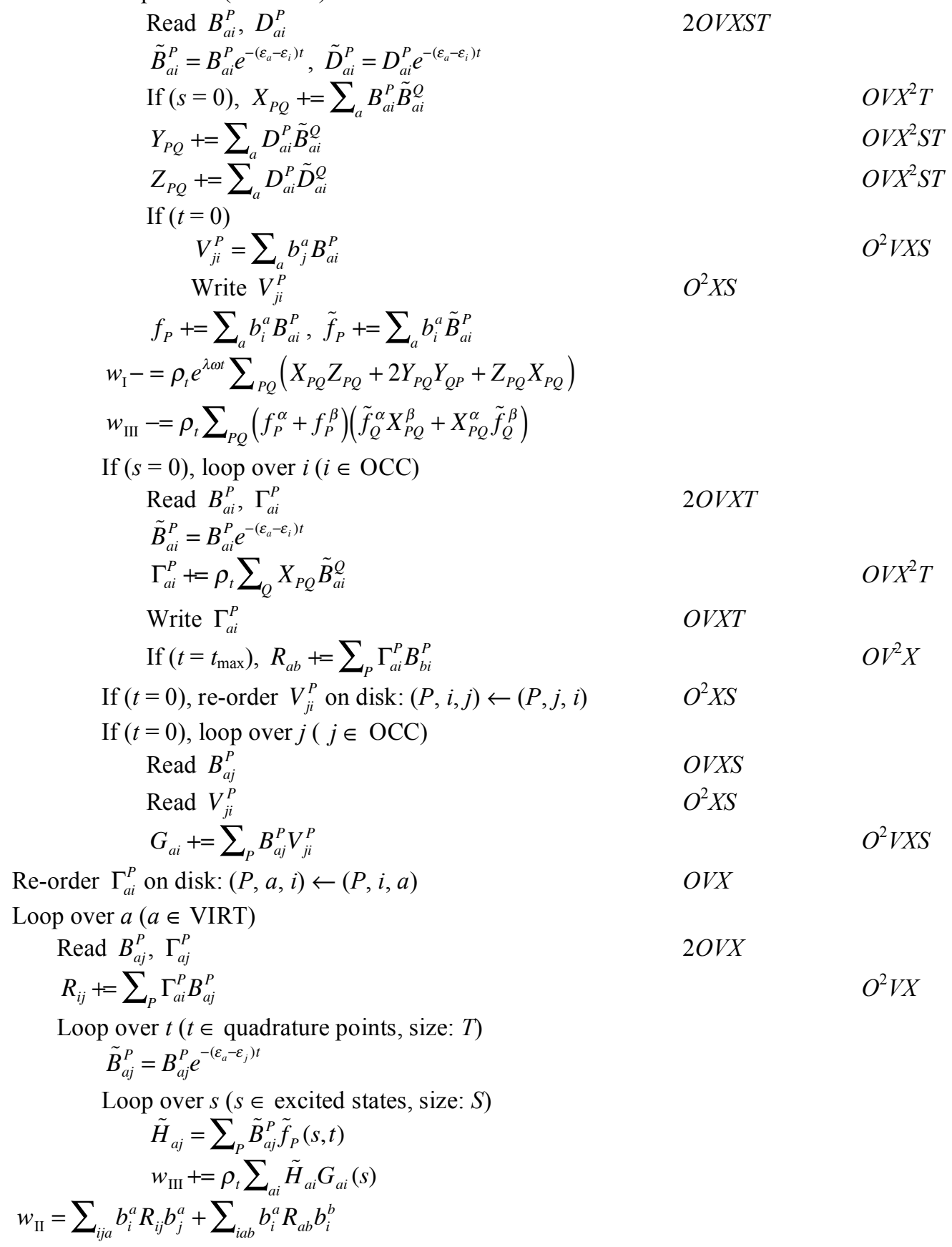

Figure 2. Algorithm for SOS-CIS(D) theory and its cost. For CPU cost, only fourth order scaling routines are noted. For visual clarity, spin designations are omitted except on Line 10 (See text for detailed equations with spin designations). 


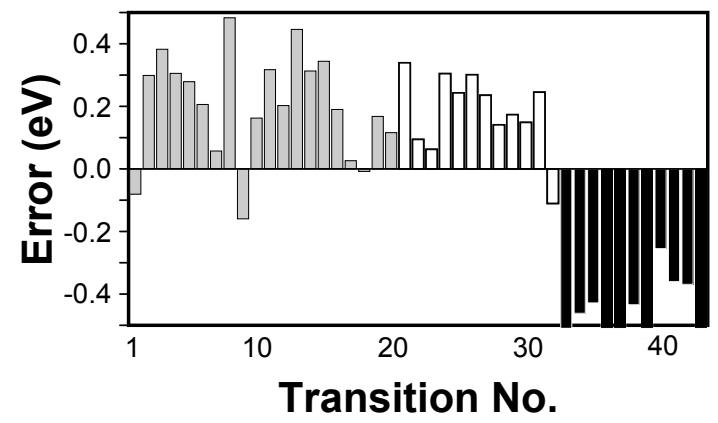

Figure 3. Errors of RI-CIS(D) with respect to experimental transition energies for $\pi \rightarrow$ $\pi^{*}$ (gray bars), $n \rightarrow \pi^{*}$ (white bars), and Rydberg (black bars) transitions. The transition numbers are listed in Table 1. 


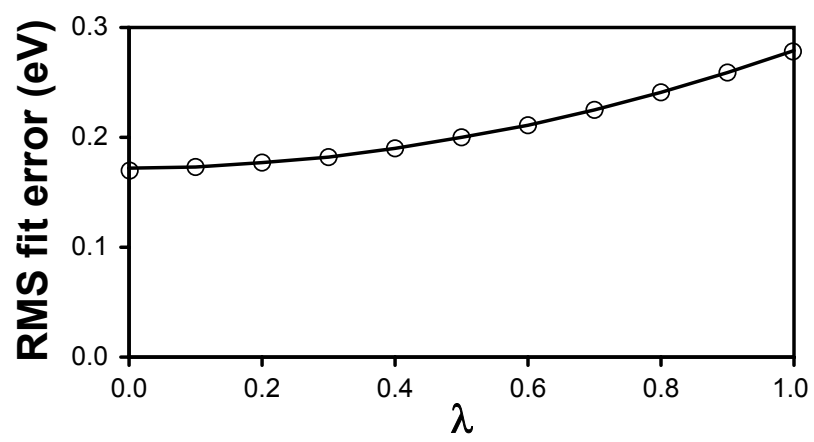

Figure 4. The variation of RMS fit error in SOS-CIS(D) as a function of damping parameter $\lambda$. 


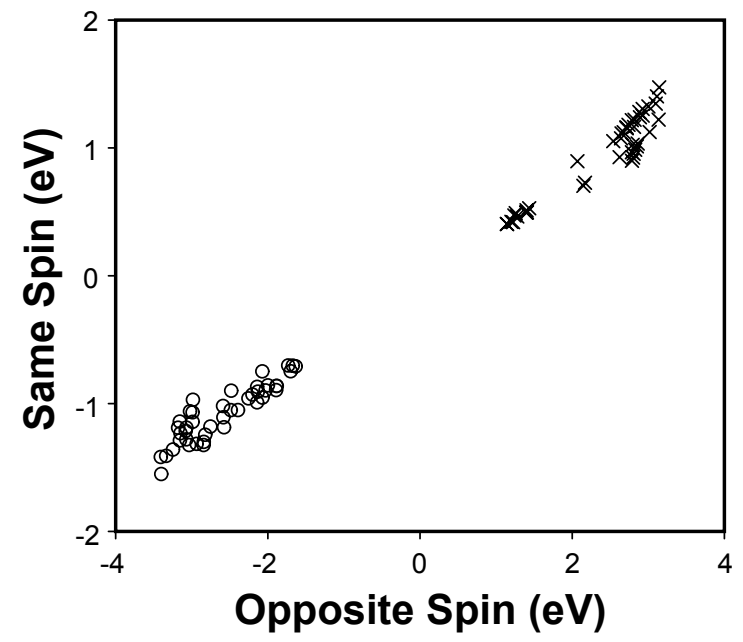

Figure 5. Correlations between same-spin and opposite-spin components in the direct term ( $w_{U}^{\mathrm{sS}}$ versus $w_{U}^{\mathrm{os}}$, marked with $\left.\mathrm{O}\right)$ and in the indirect term ( $w_{T}^{\mathrm{sS}}$ versus $w_{T}^{\mathrm{os}}$, marked with $\times)$. 

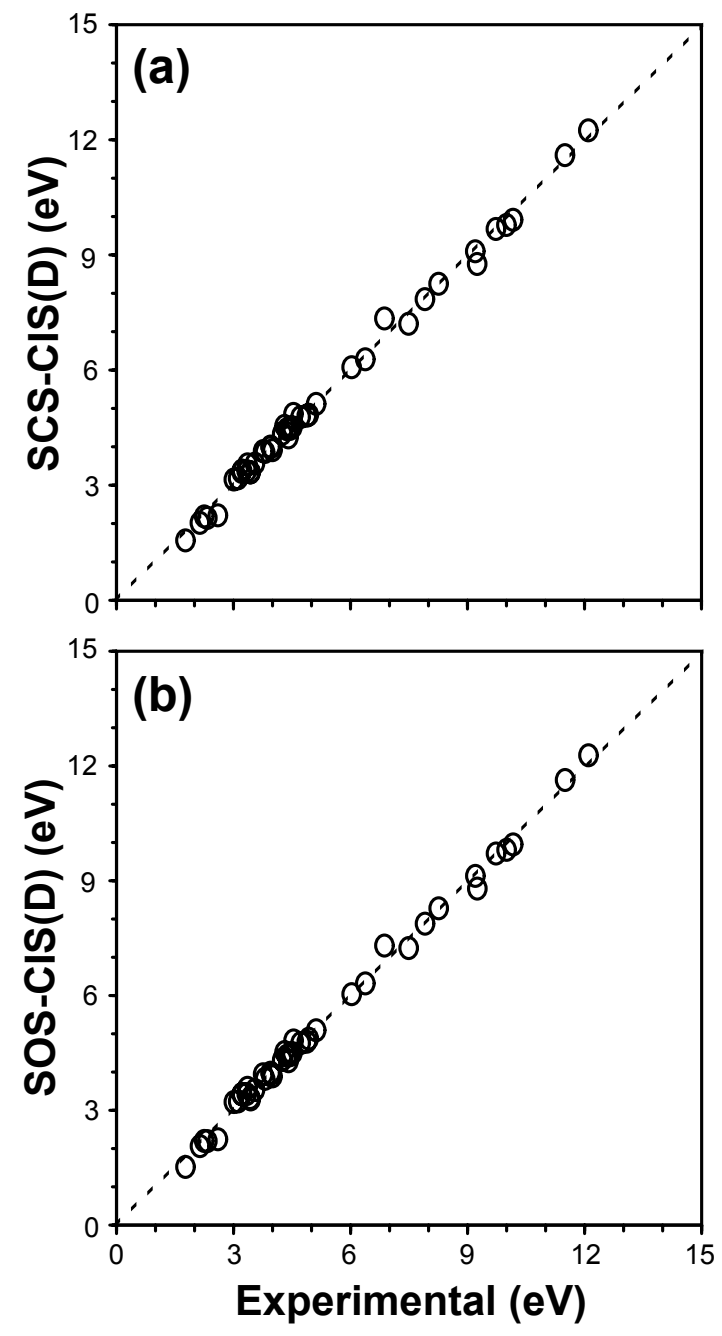

Figure 6. Correlations of transition energies from experiments and (a) SCS-CIS(D) and (b) SOS-CIS(D) theories for the molecules in the training set. The dotted line represents an ideal correlation line with slope 1 . 


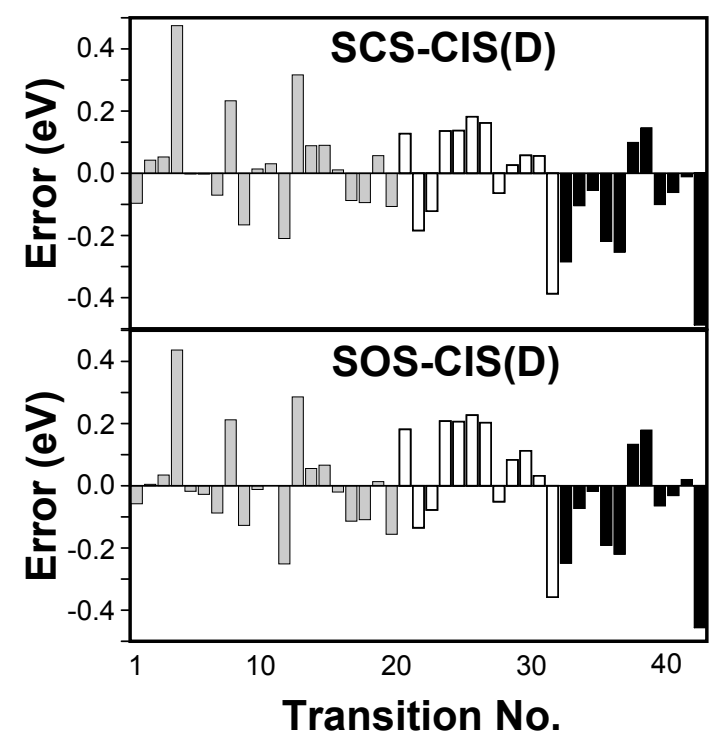

Figure 7. Errors of SCS-CIS(D) and SOS-CIS(D) with respect to experimental transition energies for $\pi \rightarrow \pi^{*}$ (gray bars), $n \rightarrow \pi^{*}$ (white bars), and Rydberg (black bars) transitions. The transition numbers are listed in Table 1. 

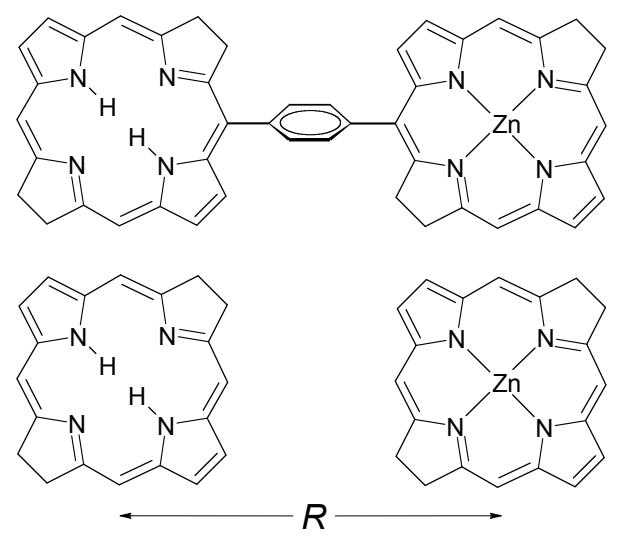

Figure 8. ZnBC-BC complex (upper panel) and its model (lower panel). The model is generated by individually aligning $\mathrm{ZnBC}$ and $\mathrm{BC}$ molecules to the matching part within the complex. $R$ denotes the centroid-to-centroid distance. $\Delta R$ is its displacement, with zero displacement defined as the distance at the original phenylene-linked complex. At zero displacement, the distance is given as $R=12.8 \AA$ with the closest hydrogen atom pair separated by $3.76 \AA$. Geometries of $\mathrm{ZnBC}-\mathrm{BC}, \mathrm{ZnBC}$, and $\mathrm{BC}$ were taken from ref 11. 


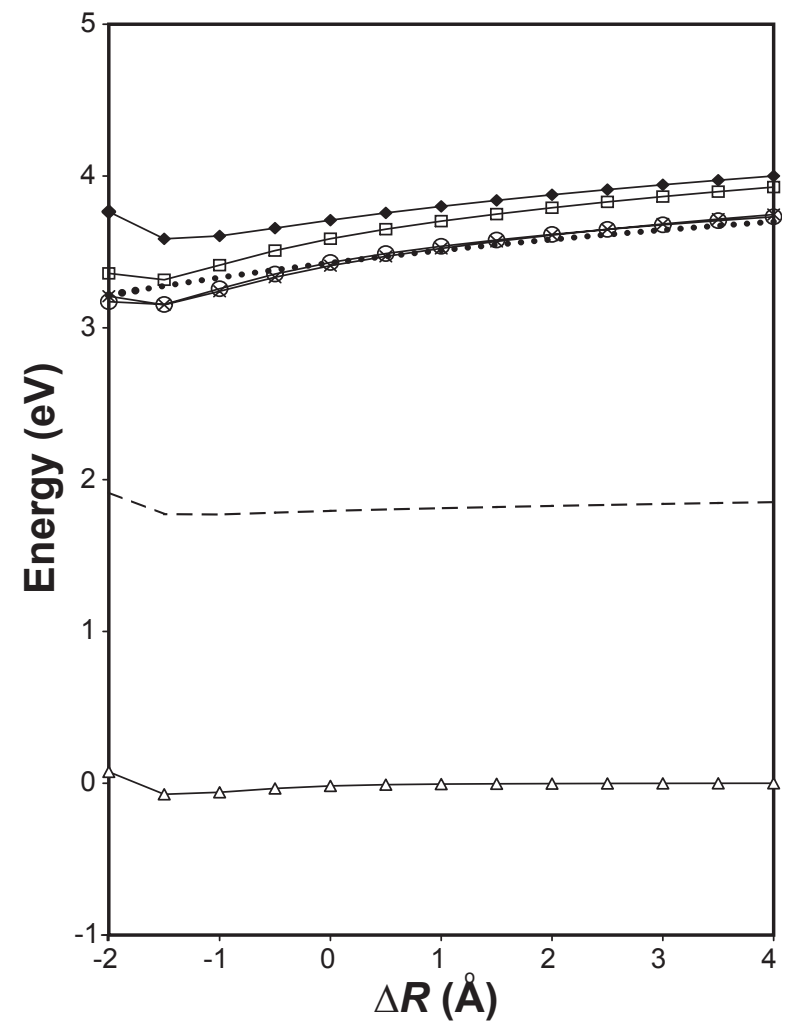

Figure 9. Potential energy curves of the lowest singlet charge transfer states of the ZnBC-BC complex model calculated with SCS-CIS(D) ( $\square$ ), SOS-CIS(D) ( $\times$ ), RI-CIS(D) $(\mathrm{O})$, CIS ( $\bullet$ ), and TDDFT (--). The ground state curve is also shown $(\triangle)$ as obtained from SOS-MP2. $\Delta R$ is explained in Figure 8 with the model complex. The dotted line shows the Coulombic interaction curve, $f(R)=-e^{2} /\left(4 \pi \varepsilon_{0} R\right)$ without any fitting (only shifted vertically so that the asymptotic vertical transition energy is the same as the difference between the ionization energy of $\mathrm{ZnBC}$ and the electron affinity of $\mathrm{BC}$ ). 


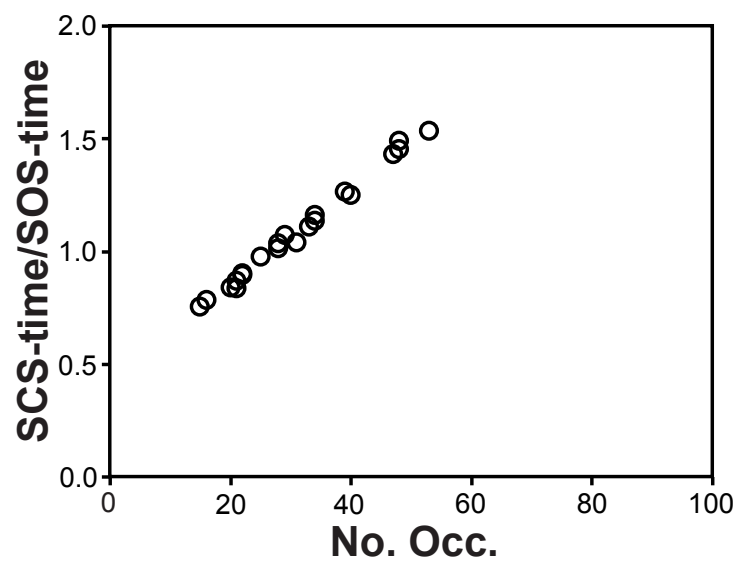

Figure 10. Ratio of CPU times of fifth order scaling SCS-CIS(D) and fourth order scaling SOS-CIS(D) as a function of the number of occupied orbitals. The organic molecules listed in Table 1 were used with 10 singlet excited state calculations. CPU time for convergence of self-consistent field (SCF) and CIS amplitude iterations is omitted in the estimation of the ratio. 